M. Nakai

Nagoya Math. J.

Vol. 186 (2007), 1-27

\title{
THE DEPENDENCE OF CAPACITIES ON MOVING BRANCH POINTS
}

\author{
MITSURU NAKAI
}

\begin{abstract}
We are concerned with the question how the capacity of the ideal boundary of a subsurface of a covering Riemann surface over a Riemann surface varies according to the variation of its branch points. In the present paper we treat the most primitive but fundamental situation that the covering surface is a two sheeted sphere with two branch points one of which is fixed and the other is moving and the subsurface is given as the complement of two disjoint continua each in different sheets of the covering surface whose projections are two disjoint continua in the base plane given in advance not touching the projections of branch points. We will derive a variational formula for the capacity and as one of its many useful consequences expected we will show that the capacity changes smoothly as one branch point moves in the subsurface.
\end{abstract}

\section{$\S 1$. Introduction}

Take a simple arc $\gamma$ on the complex sphere $\widehat{\mathbb{C}}$ connecting the point at infinity $\infty$ with a point $w$ on the complex plane $\mathbb{C}=\widehat{\mathbb{C}} \backslash\{\infty\}$. Pasting two copies of $\widehat{\mathbb{C}} \backslash \gamma$ crosswise along the arc $\gamma$ gives rise to a two sheeted sphere $\widehat{\mathbb{C}}_{\gamma}$, i.e. the covering Riemann surface $\widehat{\mathbb{C}}_{\gamma}$, or more precisely $\left(\widehat{\mathbb{C}}_{\gamma}, \widehat{\mathbb{C}}, \pi_{\gamma}\right)$, over the base surface $\widehat{\mathbb{C}}$ with the natural projection $\pi_{\gamma}$ having two and only two branch points of multiplicity 2 over $\infty$ and $w$, which we denote, as was done in $[10]$, by

$$
\widehat{\mathbb{C}}_{\gamma}:=(\widehat{\mathbb{C}} \backslash \gamma) \bigotimes_{\gamma}(\widehat{\mathbb{C}} \backslash \gamma),
$$

i.e., in general, the Riemann surface $R$ which is obtained by pasting two Riemann surfaces $S$ and $T$ crosswise along the common simple arc $\gamma$ in $S$ and $T$ in a natural sense is denoted by

$$
R:=(S \backslash \gamma) \bigotimes_{\gamma}(T \backslash \gamma) .
$$

Conformally $\widehat{\mathbb{C}}_{\gamma}$ is identical with $\widehat{\mathbb{C}}$ no matter how we choose $\gamma$. Usually $\gamma$ is referred to as the pasting arc in $\widehat{\mathbb{C}}_{\gamma}$.

Received August 2, 2005.

2000 Mathematics Subject Classification: Primary 31A15; Secondary 30C85, 30F15. 
The essential part of the result in this paper is valid for any pair of two disjoint compact subsets $A$ and $B$ in $\mathbb{C}$ but, only for the sake of simplicity, we choose and then fix two disjoint nonempty compact subsets $A$ and $B$ in $\mathbb{C}$ such that all of $\widehat{\mathbb{C}} \backslash A, \widehat{\mathbb{C}} \backslash B$, and $\widehat{\mathbb{C}} \backslash(A \cup B)$ are regular subregions of $\widehat{\mathbb{C}}$ and next take admissible arcs $\gamma$ 's in $\widehat{\mathbb{C}} \backslash(A \cup B)$ (i.e. simple arcs $\gamma$ 's in $\widehat{\mathbb{C}} \backslash(A \cup B)$ starting from $\infty$ and terminating at some points $w$ in $\mathbb{C} \backslash(A \cup B)$, which we will be calling in the sequel pasting arcs for $A \cup B)$. Embed $A$ and $B$ in $\widehat{\mathbb{C}}_{\gamma}$ in such a way that $A$ and $B$ are contained in different sheets of $\widehat{\mathbb{C}}_{\gamma}$ which are copies of $\widehat{\mathbb{C}} \backslash \gamma$ so that we understand that

$$
\left.\widehat{\mathbb{C}}_{\gamma} \backslash(A \cup B)=(\widehat{\mathbb{C}} \backslash(A \cup \gamma)) \biguplus\right\rfloor_{\gamma}(\widehat{\mathbb{C}} \backslash(B \cup \gamma))
$$

The arc $\gamma$ above in $\widehat{\mathbb{C}} \backslash(A \cup B)$ is referred to as the pasting arc for $A$ and $B$. Different from the case of $\widehat{\mathbb{C}}_{\gamma}$ the conformal structure of $\widehat{\mathbb{C}}_{\gamma} \backslash(A \cup B)$ may vary from one to other by the choice of $\gamma$ in $\widehat{\mathbb{C}} \backslash(A \cup B)$ depending upon the homotopy class in the surface $\widehat{\mathbb{C}} \backslash(A \cup B)$ to which $\gamma$ belongs.

The capacity $\operatorname{cap}\left(A, \widehat{\mathbb{C}}_{\gamma} \backslash B\right)$, or more precisely the variational 2-capacity with respect to the conformal structure of $\widehat{\mathbb{C}}_{\gamma} \backslash(A \cup B)$ is given by

$$
\operatorname{cap}\left(A, \widehat{\mathbb{C}}_{\gamma} \backslash B\right):=\inf _{\varphi} \int_{\widehat{\mathbb{C}}_{\gamma}} d \varphi \wedge * d \varphi
$$

Here the infimum above is taken with respect to the functions $\varphi$ in $C\left(\widehat{\mathbb{C}}_{\gamma}\right) \cap$ $L^{1,2}\left(\widehat{\mathbb{C}}_{\gamma}\right)$ with $\varphi \mid A=1$ and $\varphi \mid B=0$, where $L^{1,2}$ is the space of local Sobolev functions with square integrable gradients. We are interested in the variation of the capacity $\operatorname{cap}\left(A, \widehat{\mathbb{C}}_{\gamma} \backslash B\right)$ when the end point $w$ of $\gamma$, the projection of the branch point $\tilde{w}$ over $w$ varies. We may also understand that the movement of $\tilde{w}$ is described in terms of the projection $w$. In this sense the variation of the capacity with respect to $w$ is viewed as that with respect to the moving branch point $\tilde{w}$. As the central achivement of this paper we will obtain, what we call, the variational formula for the capacity, i.e. a formula computing the directional derivative

$$
\begin{aligned}
& \mathcal{D}_{w, \theta} \operatorname{cap}\left(A, \widehat{\mathbb{C}}_{\gamma} \backslash B\right) \\
& :=\lim _{r \downarrow 0} \frac{\operatorname{cap}\left(A, \widehat{\mathbb{C}}_{\gamma+\left[w, w+r e^{i \theta}\right]} \backslash B\right)-\operatorname{cap}\left(A, \widehat{\mathbb{C}}_{\gamma} \backslash B\right)}{r},
\end{aligned}
$$

of the local function $w \rightarrow \operatorname{cap}\left(A, \widehat{\mathbb{C}}_{\gamma} \backslash B\right)$ at $w \in \mathbb{C} \backslash(A \cup B)$ in the direction of $\theta$, where $\left[w, w+r e^{i \theta}\right]$ is the line segment connecting two points $w$ and 
$w+r e^{i \theta}$ (cf. the formula (2.5) in Section 2 below). Here the reason why we call $w \rightarrow \operatorname{cap}\left(A, \widehat{\mathbb{C}}_{\gamma} \backslash B\right)$ a local function is that it depends not only upon $w$ but also upon the homotopy class of $\gamma$ in $\widehat{\mathbb{C}} \backslash(A \cup B)$ and it is merely a single valued function only locally, i.e. only in each disc contained in $\mathbb{C} \backslash(A \cup B)$ centered in each point of $\mathbb{C} \backslash(A \cup B)$. Since $\widehat{\mathbb{C}}_{\gamma^{\prime}}$ only depends upon the homotopy class $\left[\gamma^{\prime}\right]$ in $\widehat{\mathbb{C}} \backslash(A \cup B)$ containing a general pasting arc $\gamma^{\prime}$ for $A$ and $B$, we may assume that $\gamma \cap\left[w, w+r e^{i \theta}\right]=\{w\}$ for all small $r>0$ so that $\gamma+\left[w, w+r e^{i \theta}\right]$ is eligible to be a pasting arc for $A$ and $B$ by deforming $\gamma$ in the homotopy class $[\gamma]$, if necessary, and thus (1.4) is well defined.

One of our original intention of the derivation of the above variational formula for the capacity, in addition to the general concern to the variational question for its own sake, is to apply it to add as many illustrative examples as possible to the classical and modern type problem (cf. e.g. [12], [17], [15], [9], [10], [6], [7], [8], etc.). However in the present paper we only state the following direct consequence of the formula as a byproduct:

TheOREM 1.1. The capacity $\operatorname{cap}\left(A, \widehat{\mathbb{C}}_{\gamma} \backslash B\right)$ varies smoothly with respect to the movement of the branch point $\tilde{w}$ of the covering surface $\widehat{\mathbb{C}}_{\gamma}$ over the end point $w$ of $\gamma$ contained in $\widehat{\mathbb{C}} \backslash(A \cup B)$, i.e. the local function $w \mapsto \operatorname{cap}\left(A, \widehat{\mathbb{C}}_{\gamma} \backslash B\right)$ is of class $C^{1}$ when $w$ varies in $\widehat{\mathbb{C}} \backslash(A \cup B)$.

We briefly describe the content of this paper. Finishing Introduction in this present Section 1 we turn to Section 2: The main result, in which we state our main result: Theorem 2.1 giving the formula of the directional derivative $\mathcal{D}_{w, \theta} \operatorname{cap}\left(A, \widehat{\mathbb{C}}_{\gamma} \backslash B\right)$ of the local function $w \mapsto \operatorname{cap}\left(A, \widehat{\mathbb{C}}_{\gamma} \backslash B\right)(w$ being the end point of $\gamma)$ at the point $w \in \mathbb{C} \backslash(A \cup B)$ in the direction $\theta$. In Section 3: Reduction to the case of direction 0, it is shown that the proof of Theorem 2.1 can be reduced to the case $\theta=0$. The proof for Theorem 2.1 reduced to the case $\theta=0$ will be given in Sections 49. The capacity $\operatorname{cap}\left(A, \widehat{\mathbb{C}}_{\gamma} \backslash B\right)$ is given by the Dirichlet integral $D\left(u_{\gamma}\right)=$ $D\left(u_{\gamma} ; \widehat{\mathbb{C}}_{\gamma} \backslash(A \cup B)\right)$ of the capacity function $u_{\gamma}$ for $\operatorname{cap}\left(A, \widehat{\mathbb{C}}_{\gamma} \backslash B\right)$ and on using the Green formula the computation concermimg $D\left(u_{\gamma}\right)$ is transformed to certain line integrals. These facts will be stated in Section 4: Prelude to the proof. We use the so called standard local parameters on $\widehat{\mathbb{C}}_{\gamma}$ and in particular at the branch points of $\widehat{\mathbb{C}}_{\gamma}$. We explain in Section 5: Standard local parameter at the branch point, how these parameters are related to each other when the end point $w$ of $\gamma$ varies. These are compiled into, what we call, a coordinate transformation formula (5.5), an essence of which 
and some related considerations are already found in the pioneering work of Rauch [13] and also in its important generalization of Yamaguchi [18] except for our present detailed examination of directional variations of capacities by the movement of branch points to clarify which is one of our objects in the present paper. We need to use the Cauchy estimates for harmonic functions in Sections 7-10 so that just for the sake of convenience we inserted here the simple and elementary Section 6: The harmonic version of the Cauchy estimates. The computations with estimates of the average rate of change $\left(D\left(u_{\mu}\right)-D\left(u_{\lambda}\right)\right) /(\mu-\lambda)$ will be done in Section 7: Calculation of $D\left(u_{\lambda}-u_{\mu}, u_{\lambda}\right)$, and Section 8: Calculation of $D\left(u_{\mu}-u_{\lambda}, u_{\lambda}\right)$. The proof of the main theorem, Theorem 2.1, will be completed in Section 9: Continuity of standard partial derivatives. The result in Section 9 is again also used in the final Section 10: Proof of Theorem 1.1.

We are grateful to Professor Hiroshi Yamaguchi for his kind concern, interest, and advice to our present work, which is closely related to a series of the important works on variation of the Robin function and so forth by Yamaguchi himself and with his colleagues (cf. e.g. [18], [19], [3], [4], [5], among many others).

\section{$\S 2$. The main result}

The variational problem (1.3) has a unique extremal function $u_{\gamma}$ in the class of competing functions $\varphi \in C\left(\widehat{\mathbb{C}}_{\gamma}\right) \cap L^{1,2}\left(\widehat{\mathbb{C}}_{\gamma}\right)$ with $\varphi \mid A=1$ and $\varphi \mid B=$ 0 in (1.3) characterized by the condition that $u_{\gamma} \in C\left(\widehat{\mathbb{C}}_{\gamma}\right) \cap H\left(\widehat{\mathbb{C}}_{\gamma} \backslash(A \cup B)\right)$ and $u_{\gamma} \mid A=1$ and $u_{\gamma} \mid B=0$ (cf. e.g. [15], [2], etc.), where in general we denote by $H(X)$ the class of harmonic functions defined on an open subset $X$ of some Riemann surface and $L^{1,2}$ indicates the Dirichlet space (cf. [2], and also the paragraph following the formula (1.3) below). The function $u_{\gamma}$ is referred to as the capacity function ([14]) for $A$ with respect to $\widehat{\mathbb{C}}_{\gamma} \backslash B$ and $u_{\gamma} \mid\left(\widehat{\mathbb{C}}_{\gamma} \backslash(A \cup B)\right)$ is called the harmonic measure of $\partial A$ with respect to $\widehat{\mathbb{C}}_{\gamma} \backslash(A \cup B)$. Then the capacity $\operatorname{cap}\left(A, \widehat{\mathbb{C}}_{\gamma} \backslash B\right)$ is given by

$$
\operatorname{cap}\left(A, \widehat{\mathbb{C}}_{\gamma} \backslash B\right)=\int_{\widehat{\mathbb{C}}_{\gamma}} d u_{\gamma} \wedge * d u_{\gamma}=\int_{\widehat{\mathbb{C}}_{\gamma} \backslash(A \cup B)} d u_{\gamma} \wedge * d u_{\gamma}
$$

in terms of the capacity function. When we wish to stress that the end point of $\gamma$ is $w$, we write $\widehat{\mathbb{C}}_{\gamma}=\widehat{\mathbb{C}}_{\gamma, w}$ and similarly $u_{\gamma}=u_{\gamma, w}$ indicating the point $w$ and moreover when $\gamma$ is well understood, we simply write $\widehat{\mathbb{C}}_{\gamma}=\widehat{\mathbb{C}}_{w}$ and $u_{\gamma}=u_{w}$. 
Let $z$ be the proper plane coordinate in $\mathbb{C}$. Now we introduce the most primitive and simple local parameters on the two sheeted sphere $\widehat{\mathbb{C}}_{\gamma}$ determined by the pasting arc $\gamma$ connecting the point $\infty$ at infinity and a finite point $w \in \mathbb{C}$. We denote by $\Delta(a, r)$ the open disc with radius $r>0$ centered at $a \in \mathbb{C}$ and by $\Delta(\infty, r)$ the open $\operatorname{disc}\{z \in \mathbb{C}:|z|>1 / r\} \cup\{\infty\}$. First pick a ordinary point $p \in \widehat{\mathbb{C}}_{\gamma} \backslash\{\tilde{\infty}, \tilde{w}\}\left(\pi_{\gamma}(\tilde{\infty})=\infty, \pi_{\gamma}(\tilde{w})=w\right)$ and a $\operatorname{disc} \Delta\left(\pi_{\gamma}(p), r\right) \subset \mathbb{C} \backslash\{\infty, w\}\left(0<r<\left|w-\pi_{\gamma}(p)\right|\right)$. We take $\left(\Delta\left(\pi_{\gamma}(p), r\right), \zeta\right)$ as a parametric disc at $p$, where the local parameter $\zeta=\xi+i \eta$ at $p$ is given by

$$
\zeta=z-\pi_{\gamma}(p) .
$$

At the finite branch point $\tilde{w}$ over $w \in \mathbb{C}$, we take $(\Delta(w, r), \zeta)(r>0)$ as a parametric disc at $\tilde{w}$, where the local parameter $\zeta=\xi+i \eta$ at $\tilde{w}$ is given by

$$
\zeta=\sqrt{z-w} \quad(\sqrt{1}=1) .
$$

Finally at the other branch point $\tilde{\infty}$ over $\infty \in \widehat{\mathbb{C}}$ we take $(\Delta(\infty, r), \zeta)$ $(0<r<1 /|w|)$ as a parametric disc at $\tilde{\infty}$, where the local parameter $\zeta=\xi+i \eta$ at $\tilde{\infty}$ is given by

$$
\zeta=\sqrt{1 / z} \quad(\sqrt{1 / c}>0)
$$

for every real number $0<c<|w|$. The local parameter $\zeta=\xi+i \eta$ defined by $(2.2),(2.3)$, and (2.4) is referred to as the standard local parameters in $\widehat{\mathbb{C}}_{\gamma}$.

Let $w \in \mathbb{C}$ and $\theta \in \mathbb{R}$. Using the capacity function $u_{\gamma, w}$ and the standard local parameter $\zeta=\xi+i \eta$ in (2.3) introduced above we can state the following variational formula for the capacity, which is the main result of this paper.

TheOREm 2.1. The directional derivative $\mathcal{D}_{w, \theta} \operatorname{cap}\left(A, \widehat{\mathbb{C}}_{\gamma, w} \backslash B\right)$ of the capacity $\operatorname{cap}\left(A, \widehat{\mathbb{C}}_{\gamma, w} \backslash B\right)$ at $w$ in the direction of $\theta$ is given by

$$
\begin{aligned}
\mathcal{D}_{w, \theta} \operatorname{cap}\left(A, \widehat{\mathbb{C}}_{\gamma, w} \backslash B\right)=\pi[ & \left(\left(\frac{\partial}{\partial \xi} u_{\gamma, w}(0)\right)^{2}-\left(\frac{\partial}{\partial \eta} u_{\gamma, w}(0)\right)^{2}\right) \cos \theta \\
& \left.+2\left(\frac{\partial}{\partial \xi} u_{\gamma, w}(0) \cdot \frac{\partial}{\partial \eta} u_{\gamma, w}(0)\right) \sin \theta\right]
\end{aligned}
$$


In the particular case of the direction $\theta=0$, the above formula takes the following simple form:

$$
\mathcal{D}_{w, 0} \operatorname{cap}\left(A, \widehat{\mathbb{C}}_{\gamma, w} \backslash B\right)=\pi\left[\left(\frac{\partial}{\partial \xi} u_{\gamma, w}(0)\right)^{2}-\left(\frac{\partial}{\partial \eta} u_{\gamma, w}(0)\right)^{2}\right] .
$$

We will first prove in Section 3 that conversely (2.6) implies (2.5), i.e. the reduction of (2.5) to (2.6) so that we only have to concentrate ourselves to the proof of (2.6) in the rest of our paper in Sections 4-9.

We add one more remark to the expression (2.5). We consider the gradient of $u_{\gamma, w}$ with respect to the standard local parameter $\zeta$ in $(2.3)$ at $w$ considered for $\zeta=0: \nabla_{\zeta} u_{\gamma, w}(0)=\left(\partial u_{\gamma, w}(0) / \partial \xi, \partial u_{\gamma, w}(0) / \partial \eta\right)=$ $\left.\left(\partial u_{\gamma, w}(\zeta) / \partial \xi, \partial u_{\gamma, w}(\zeta) / \partial \eta\right)\right|_{\zeta=0}$. The angle

$$
\alpha_{\gamma, w}:=\tan ^{-1} \frac{2\left(\partial u_{\gamma, w}(0) / \partial \xi\right) \cdot\left(\partial u_{\gamma, w}(0) / \partial \eta\right)}{\left(\partial u_{\gamma, w}(0) / \partial \xi\right)^{2}-\left(\partial u_{\gamma, w}(0) / \partial \eta\right)^{2}} \in[-\pi / 2, \pi / 2]
$$

is referred to as the direction of maximal growth of cap $\left(A, \widehat{\mathbb{C}}_{\gamma, w} \backslash B\right)$ at $w$. Then it can be really easily seen that $(2.5)$ is rewritten as follows:

$$
\mathcal{D}_{w, \theta} \operatorname{cap}\left(A, \widehat{\mathbb{C}}_{\gamma, w} \backslash B\right)=\left|\nabla_{\zeta} u_{\gamma, w}(0)\right|^{2} \cos \left(\theta-\alpha_{\gamma, w}\right),
$$

which is sometimes very handy to apply to various practical situations.

\section{§3. Reduction to the case of direction zero}

We prove that the validity of (2.6) implies that of (2.5). We denote by $(\widehat{\mathbb{C}}, z)$ the sphere with a proper plane coordinate $z$ on $\widehat{\mathbb{C}}$. If we obtain another proper plane coordinate $\tilde{z}$ by applying a translation and rotation to $z$, then we also obtain the sphere $(\widehat{\mathbb{C}}, \tilde{z})$ with a proper plane coordinate $\tilde{z}$. Precisely we consider the new coordinate $\tilde{z}$ obtained from the original coordinate $z$ by the transformation

$$
\tilde{z}-w=(z-w) e^{i \theta}
$$

We denote by $\gamma, w$ the pasting arc $\gamma$ indicating and also stressing its terminal point $w$. Then, although the pasting arc $\gamma, w$ itself is unchanged no matter whether it is in $(\widehat{\mathbb{C}}, z)$ or in $(\widehat{\mathbb{C}}, \tilde{z})$, its coordinate expression in $(\widehat{\mathbb{C}}, \tilde{z})$ is different from that of the original one in $(\widehat{\mathbb{C}}, z)$ and hence we denote $\gamma, w$ by $\tilde{\gamma}, w$ in $(\widehat{\mathbb{C}}, \tilde{z})$. Of course

$$
\operatorname{cap}\left(A, \widehat{\mathbb{C}}_{\gamma, w} \backslash B\right)=\operatorname{cap}\left(A, \widehat{\mathbb{C}}_{\tilde{\gamma}, w} \backslash B\right)
$$


and their capacity functions are identical. That of the former has been denoted by $u_{\gamma, w}$ and now we denote the capacity function of the latter above by $\tilde{u}_{\tilde{\gamma}, w}$ for the reason similar to the case of $\gamma$. Finally we denote by $\tilde{\mathcal{D}}_{w, 0} \operatorname{cap}\left(A, \widehat{\mathbb{C}}_{\tilde{\gamma}, w} \backslash B\right)$ the directional derivative of $\operatorname{cap}\left(A, \widehat{\mathbb{C}}_{\tilde{\gamma}, w} \backslash B\right)$ at $w$ in the direction of the angle 0 :

$$
\begin{aligned}
\tilde{\mathcal{D}}_{w, 0} \operatorname{cap}\left(A, \widehat{\mathbb{C}}_{\tilde{\gamma}, w} \backslash B\right) \\
\quad=\lim _{r \downarrow 0} \frac{\operatorname{cap}\left(A, \widehat{\mathbb{C}}_{\tilde{\gamma}+[w, w+r], w+r} \backslash B\right)-\operatorname{cap}\left(A, \widehat{\mathbb{C}}_{\tilde{\gamma}, w} \backslash B\right)}{r} .
\end{aligned}
$$

The standard local parameter $\tilde{\zeta}=\tilde{\xi}+i \tilde{\eta}$ at the branch point $w$ of $\widehat{\mathbb{C}}_{\tilde{\gamma}, w}$ on it is given (cf. (2.3)) by

$$
\tilde{\zeta}=\tilde{\xi}+i \tilde{\eta}=\sqrt{\tilde{z}-w} \quad(\sqrt{1}=1) .
$$

Since we are assuming the validity of (2.6) we have

$$
\tilde{\mathcal{D}}_{w, 0} \operatorname{cap}\left(A, \widehat{\mathbb{C}}_{\tilde{\gamma}, w} \backslash B\right)=\pi\left[\left(\frac{\partial}{\partial \tilde{\xi}} \tilde{u}_{\tilde{\gamma}, w}(0)\right)^{2}-\left(\frac{\partial}{\partial \tilde{\eta}} \tilde{u}_{\tilde{\gamma}, w}(0)\right)^{2}\right] .
$$

From the coordinate transformation (3.1) we see that

$$
\mathcal{D}_{w, \theta} \operatorname{cap}\left(A, \widehat{\mathbb{C}}_{\gamma, w} \backslash B\right)=\tilde{\mathcal{D}}_{w, 0} \operatorname{cap}\left(A, \widehat{\mathbb{C}}_{\tilde{\gamma}, w} \backslash B\right) .
$$

By using (2.3), (3.2), and (3.1) in this order we have

$$
\zeta=\sqrt{z-w}=\sqrt{e^{i \theta(\tilde{z}-w)}}=e^{i \theta / 2} \sqrt{\tilde{z}-w}=e^{i \theta / 2} \tilde{\zeta}
$$

so that

$$
\left(\begin{array}{l}
\xi \\
\eta
\end{array}\right)=\left(\begin{array}{cc}
\cos (\theta / 2) & -\sin (\theta / 2) \\
\sin (\theta / 2) & \cos (\theta / 2)
\end{array}\right)\left(\begin{array}{l}
\tilde{\xi} \\
\tilde{\eta}
\end{array}\right)
$$

In view of

$$
\begin{aligned}
\frac{\partial}{\partial \tilde{\xi}} \tilde{u}_{\tilde{\gamma}, w}(0) & =\frac{\partial}{\partial \xi} u_{\gamma, w}(0) \frac{\partial \xi}{\partial \tilde{\xi}}+\frac{\partial}{\partial \eta} u_{\gamma, w}(0) \frac{\partial \eta}{\partial \tilde{\xi}} \\
& =\frac{\partial}{\partial \xi} u_{\gamma, w}(0) \cos \frac{\theta}{2}+\frac{\partial}{\partial \eta} u_{\gamma, w}(0) \sin \frac{\theta}{2}
\end{aligned}
$$

and the similar formula for $\partial \tilde{u}_{\tilde{\gamma}, w}(0) / \partial \tilde{\eta}$, we have

$$
\left(\begin{array}{l}
\partial \tilde{u}_{\tilde{\gamma}, w}(0) / \partial \tilde{\xi} \\
\partial \tilde{u}_{\tilde{\gamma}, w}(0) / \partial \tilde{\eta}
\end{array}\right)=\left(\begin{array}{cc}
\cos (\theta / 2) & \sin (\theta / 2) \\
-\sin (\theta / 2) & \cos (\theta / 2)
\end{array}\right)\left(\begin{array}{c}
\partial u_{\gamma, w}(0) / \partial \xi \\
\partial u_{\gamma, w}(0) / \partial \eta
\end{array}\right)
$$


If we substitute $\partial u_{\gamma, w}(0) / \partial \xi$ and $\partial u_{\gamma, w}(0) / \partial \eta$ in (3.3) by the above transformation formula, then we have

$$
\begin{aligned}
\tilde{\mathcal{D}}_{w, 0} \operatorname{cap}\left(A, \widehat{\mathbb{C}}_{\tilde{\gamma}, w} \backslash B\right)=\pi[ & \left(\frac{\partial}{\partial \xi} u_{\gamma, w}(0) \cos \frac{\theta}{2}+\frac{\partial}{\partial \eta} u_{\gamma, w}(0) \sin \frac{\theta}{2}\right)^{2} \\
& \left.-\left(\frac{\partial}{\partial \xi} u_{\gamma, w}(0) \cos \frac{\theta}{2}+\frac{\partial}{\partial \eta} u_{\gamma, w}(0) \sin \frac{\theta}{2}\right)^{2}\right] \\
=\pi[( & \left.\left(\frac{\partial}{\partial \xi} u_{\gamma, w}(0)\right)^{2}-\left(\frac{\partial}{\partial \eta} u_{\gamma, w}(0)\right)^{2}\right) \cos \theta \\
+ & \left.2\left(\frac{\partial}{\partial \xi} u_{\gamma, w}(0) \cdot \frac{\partial}{\partial \eta} u_{\gamma, w}(0)\right) \sin \theta\right] .
\end{aligned}
$$

This with (3.4) implies (2.5).

\section{$\S 4$. Prelude to the proof}

We now start the proof of (2.6). By applying a suitable translation of the original plane coordinate $z$ in $(\widehat{\mathbb{C}}, z)$, if necessary, we may assume that the end point $w$ of $\gamma$ in (2.6) is real, i.e. $w \in \mathbb{R}$, the real axis in $\mathbb{C}$. Changing the notation we write $w=\lambda \in \mathbb{R}$ so that we are going to prove

$$
\mathcal{D}_{\lambda, 0} \operatorname{cap}\left(A, \widehat{\mathbb{C}}_{\gamma, \lambda} \backslash B\right)=\pi\left[\left(\frac{\partial}{\partial \xi} u_{\gamma, \lambda}(0)\right)^{2}-\left(\frac{\partial}{\partial \eta} u_{\gamma, \lambda}(0)\right)^{2}\right],
$$

where $\zeta=\xi+i \eta=\sqrt{z-\lambda}(\sqrt{1}=1)$, the standard local parameter of $\widehat{\mathbb{C}}_{\gamma, \lambda}$ at the branch point $\tilde{\lambda}$ over $\lambda$.

At this point it is convenient to introduce the following notations. For $\varphi$ and $\psi$ in $L^{1,2}(X), X$ being a measurable subset of a Riemann surface and $L^{1,2}(X)$ being the Dirichlet space (cf. [2]) on $X$ consisting of real valued functions $f \in W_{l o c}^{1,2}(X)$, the local Sobolev space on $X$, with square integrable gradients, the quantity

$$
D(\varphi, \psi ; X):=\int_{X} d \varphi \wedge * d \psi=\int_{X} \nabla \varphi(\zeta) \cdot \nabla \psi(\zeta) d \xi d \eta \quad(\zeta=\xi+i \eta)
$$

is referred to as the mutual Dirichlet integral of $\varphi$ and $\psi$ over $X$, where $\zeta=\xi+i \eta$ is any local parameter on the Riemann surface and $\nabla \varphi=\operatorname{grad} \varphi=$ $(\partial \varphi / \partial \xi, \partial \varphi / \partial \eta)$. In particular

$$
D(\varphi ; X):=\int_{X} d \varphi \wedge * d \varphi=\int_{X}|\nabla \varphi(\zeta)|^{2} d \xi d \eta \quad(\zeta=\xi+i \eta)
$$


is called the Dirichlet integral of $\varphi$ over $X$. The mutual Dirichlet integral, viewed as a mapping $(\varphi, \psi) \mapsto D(\varphi, \psi ; X): L^{1,2}(X) \rightarrow \mathbb{R}$ is a bilinear form and the square root of the Dirichlet integral $\sqrt{D(\varphi ; X)}$ is a seminorm on $L^{1,2}(X)$.

With respect to the fixed pasting arc $\gamma, \lambda$ with $\lambda \in \mathbb{R}$ in (4.1), we can choose a closed interval $[\sigma, \tau]:=\{t \in \mathbb{R}: \sigma \leq t \leq \tau\}(\sigma<\lambda<\tau)$ such that we may assume the following three conditions:

(a) the straight line segment $[\sigma, \tau]$ is contained in $\mathbb{C} \backslash(A \cup B)$;

(b) the straight line segment $[\sigma, \lambda]$ is an end part of $\gamma$, i.e. the subarc of $\gamma$ starting from the point $\sigma$ in $\gamma$ and terminating at $\lambda$;

(c) $\gamma \cap[\lambda, \tau]=\{\lambda\}$ so that the arc $\gamma+[\lambda, \tau]$ is a pasting arc for $A$ and $B$.

In fact, if we choose $\sigma<\lambda$ and $\tau>\lambda$ in $\mathbb{R}$ close enough to $\lambda$, then we can find a pasting arc $\gamma^{\prime}, \lambda$ for $A$ and $B$ homotopic to $\gamma$ in $\widehat{\mathbb{C}} \backslash(A \cup B)$ satisfying three conditions (a), (b), and (c) simultaneously. By the homotopical invariance of the conformal structure of $\widehat{\mathbb{C}}_{\gamma, \lambda} \backslash(A \cup B)$ for $\gamma \subset \widehat{\mathbb{C}} \backslash(A \cup B)$, we can replace $\gamma$ by $\gamma^{\prime}$ in (4.1) so that we may assume that $\gamma$ satisfies (a), (b), and (c) in advance.

Now we choose a real positive number $0<r<1$ so small that

$$
\sigma<\lambda-4 r^{2}<\lambda+8 r^{2}<\tau
$$

and, that if we denote by $\gamma_{\sigma}$ the subarc of $\gamma$ starting from $\infty$ and terminating at $\sigma$, then

$$
\operatorname{dis}\left(\left[\lambda-4 r^{2}, \lambda+8 r^{2}\right], A \cup B \cup \gamma_{\sigma}\right)>4 r^{2},
$$

where $\operatorname{dis}(X, Y)$ for subsets $X$ and $Y$ in $\mathbb{C}$ is the distance between $X$ and $Y$ measured by the Euclidean plane metric on $\mathbb{C}$. After we have fixed the interval $[\sigma, \tau]$ and the positive number $r>0$ as above we take a variable number $\mu \in \mathbb{R}$ satisfying

$$
\lambda \leq \mu<\lambda+r^{2}
$$

and we consider the pasting arc $\gamma(\mu)$ for $A$ and $B$ given by

$$
\gamma(\mu):=\gamma+[\lambda, \mu]
$$

so that $\gamma(\mu)=\gamma(\mu), \mu$ and $\gamma(\lambda)=\gamma=\gamma, \lambda$ in the former notation. Let $\widehat{\mathbb{C}}_{j}$ $(j=1,2)$ be two copies of $\widehat{\mathbb{C}}$ and we set

$$
\widehat{\mathbb{C}}_{\mu}:=(\widehat{\mathbb{C}} \backslash \gamma(\mu)) \bigcup_{\gamma(\mu)}(\widehat{\mathbb{C}} \backslash \gamma(\mu))=\left(\widehat{\mathbb{C}}_{1} \backslash \gamma(\mu)\right) \bigcup \bigcup_{\gamma(\mu)}\left(\widehat{\mathbb{C}}_{2} \backslash \gamma(\mu)\right)
$$


so that $\widehat{\mathbb{C}}_{\mu}=\widehat{\mathbb{C}}_{\gamma(\mu)}=\widehat{\mathbb{C}}_{\gamma(\mu), \mu}$ in the former notation. Recall that $A \subset \widehat{\mathbb{C}}_{1}$ and $B \subset \widehat{\mathbb{C}}_{2}$, i.e. $A$ and $B$ are embeded in the different sheets of $\widehat{\mathbb{C}}_{\mu}$.

Finally let $u_{\mu}:=u_{\gamma(\mu), \mu}$ be the capacity function for $\operatorname{cap}\left(A, \widehat{\mathbb{C}}_{\mu} \backslash B\right)=$ $\operatorname{cap}\left(A, \widehat{\mathbb{C}}_{\gamma(\mu), \mu} \backslash B\right)$ so that

$$
\operatorname{cap}\left(A, \widehat{\mathbb{C}}_{\mu} \backslash B\right)=\int_{\widehat{\mathbb{C}}_{\mu}} d u_{\mu} \wedge * d u_{\mu}=D\left(u_{\mu} ; \widehat{\mathbb{C}}_{\mu}\right) .
$$

Hence we are going to compute

$$
\mathcal{D}_{\lambda, 0} \operatorname{cap}\left(A, \widehat{\mathbb{C}}_{\lambda} \backslash B\right)=\lim _{\mu \downarrow \lambda} \frac{D\left(u_{\mu} ; \widehat{\mathbb{C}}_{\mu}\right)-D\left(u_{\lambda} ; \widehat{\mathbb{C}}_{\lambda}\right)}{\mu-\lambda}
$$

in order to derive (4.1). Here a serious difficulty arises. The domains $\widehat{\mathbb{C}}_{\mu}$ and $\widehat{\mathbb{C}}_{\lambda}$ of $u_{\mu}$ and $u_{\lambda}$ are entirely different surfaces and hence $D\left(u_{\mu}, \widehat{\mathbb{C}}_{\mu}\right)-$ $D\left(u_{\lambda} ; \widehat{\mathbb{C}}_{\lambda}\right)$ cannot be computed any further. To overcome this difficulty we recall that $\widehat{\mathbb{C}}_{\mu}$ is a covering surface $\left(\widehat{\mathbb{C}}_{\mu}, \widehat{\mathbb{C}}, \pi_{\mu}\right)$ of the base surface $\widehat{\mathbb{C}}$ with $\pi_{\mu}$ its natural projection having two branch points $\tilde{\infty}$ over $\infty$ and $\tilde{\mu}$ over $\mu$ each of which is of multiplicity two. Observe that $\widehat{\mathbb{C}}_{\mu} \backslash \pi_{\mu}^{-1}([\lambda, \mu])$ and $\widehat{\mathbb{C}}_{\lambda} \backslash \pi_{\lambda}^{-1}([\lambda, \mu])$ may be viewed as identical simply connected region so that we denote by $W=W_{\mu}$ the common Riemann surface:

$$
W=W_{\mu}:=\widehat{\mathbb{C}}_{\mu} \backslash \pi_{\mu}^{-1}([\lambda, \mu])=\widehat{\mathbb{C}}_{\lambda} \backslash \pi_{\lambda}^{-1}([\lambda, \mu]),
$$

and moreover

$$
\pi_{\mu}\left|W=\pi_{\lambda}\right| W
$$

so that $\left(W, \widehat{\mathbb{C}} \backslash[\lambda, \mu], \pi_{\mu}\right)=\left(W, \widehat{\mathbb{C}} \backslash[\lambda, \mu], \pi_{\lambda}\right)$ as covering surfaces over $\widehat{\mathbb{C}} \backslash[\lambda, \mu]$. In particular we see that $D\left(u_{\mu} ; \widehat{\mathbb{C}}_{\mu}\right)=D\left(u_{\mu} ; W\right)$ and $D\left(u_{\lambda} ; \widehat{\mathbb{C}}_{\lambda}\right)=$ $D\left(u_{\lambda} ; W\right)$. Hereafter as far as the relation $\lambda<\mu<\lambda+r^{2}$ is retained we simply write $D(\cdot, \cdot ; W)=D(\cdot, \cdot)$ so that $D\left(u_{\mu} ; W\right)=D\left(u_{\mu}\right)$ and $D\left(u_{\lambda} ; W\right)=$ $D\left(u_{\lambda}\right)$. Then we can compute $D\left(u_{\mu} ; \widehat{\mathbb{C}}_{\mu}\right)-D\left(u_{\lambda} ; \widehat{\mathbb{C}}_{\lambda}\right)=D\left(u_{\mu}\right)-D\left(u_{\lambda}\right)$ as follows:

$$
D\left(u_{\mu}\right)-D\left(u_{\lambda}\right)=-D\left(u_{\lambda}-u_{\mu}, u_{\lambda}\right)+D\left(u_{\mu}-u_{\lambda}, u_{\mu}\right) .
$$

We are thus led to study two mutual Dirichlet integrals $D\left(u_{\lambda}-u_{\mu}, u_{\lambda}\right)$ and $D\left(u_{\mu}-u_{\lambda}, u_{\mu}\right)$.

Although $\widehat{\mathbb{C}}_{\mu} \backslash \pi_{\mu}^{-1}([\lambda, \mu])$ and $\widehat{\mathbb{C}}_{\lambda} \backslash \pi_{\lambda}^{-1}([\lambda, \mu])$ are the identical simply connected region $W$, their Carathéodory boundaries (the set of boundary 
elements in the sense of Carathéodory (cf. e.g. [17])) can be differently described as subsurfaces of $\widehat{\mathbb{C}}_{\mu}$ and $\widehat{\mathbb{C}}_{\lambda}$. We first examine $\widehat{\mathbb{C}}_{\lambda} \backslash \pi_{\lambda}^{-1}([\lambda, \mu])$. The fiber $\pi_{\lambda}^{-1}(\lambda)$ is a one point set in $\widehat{\mathbb{C}}_{\lambda}$, the point in which will be denoted by $\lambda_{0}$, and the point $\lambda_{0}$ is the branch point of multiplicity 2 of the covering surface $\left(\widehat{\mathbb{C}}_{\lambda}, \widehat{\mathbb{C}}, \pi_{\lambda}\right)$. But the fiber $\pi_{\lambda}^{-1}(\mu)$ is a two point set consisting of a point $\mu_{1}$ in the upper sheet $\widehat{\mathbb{C}}_{1} \backslash \gamma(\lambda)$ and a point $\mu_{2}$ in the lower sheet $\widehat{\mathbb{C}}_{2} \backslash \gamma(\lambda)$ of $\widehat{\mathbb{C}}_{\lambda}$. We denote by $a_{1}=a_{1}(\mu)$ the upper edge and by $a_{2}=a_{2}(\mu)$ the lower edge of the line segment $\pi_{\lambda}^{-1}([\lambda, \mu]) \cap \overline{\left(\widehat{\mathbb{C}}_{1} \backslash \gamma(\lambda)\right)}$, the closure being taken in $\widehat{\mathbb{C}}_{\lambda}$; similarly we denote by $a_{3}=a_{3}(\mu)$ and $a_{4}=a_{4}(\mu)$ the upper and the lower edge of the line segment $\pi_{\lambda}^{-1}([\lambda, \mu]) \cap \overline{\left(\widehat{\mathbb{C}}_{2} \backslash \gamma(\lambda)\right)}$, the closure being taken in $\widehat{\mathbb{C}}_{\lambda}$. We consider that $a_{1}$ and $a_{2}$ are directed line segments whose initial points are $\lambda_{0}$ and whose terminal points are $\mu_{1}$, respectively, and that $a_{3}$ and $a_{4}$ are also directed line segments starting from $\lambda_{0}$ and terminating at $\mu_{2}$, respectively. In general we denote by $|\omega|$ the mere point set consisting points in an arc $\omega$ in a certain Riemann surface. The set $|\omega|$ will be referred to as the support of $\omega$. Then

$$
\left|a_{1}\right|=\left|a_{2}\right|, \quad\left|a_{3}\right|=\left|a_{4}\right| .
$$

Then the Carathéodory boundary

$$
a=a(\mu):=a_{1}-a_{2}+a_{3}-a_{4}
$$

of $\widehat{\mathbb{C}}_{\lambda} \backslash \pi_{\lambda}^{-1}([\lambda, \mu])$ is a positively directed Jordan curve in the Carathéodory compactification (i.e. the union of $\widehat{\mathbb{C}}_{\lambda} \backslash \pi_{\lambda}^{-1}([\lambda, \mu])$ and $a(\mu)$ topologized suitably) of $\widehat{\mathbb{C}}_{\lambda} \backslash \pi_{\lambda}^{-1}([\lambda, \mu])$. Observe that $a \backslash\left\{\mu_{1}, \mu_{2}\right\}$ is analytic and $a$ has corners at $\mu_{1}$ and also at $\mu_{2}$ considered in $\widehat{\mathbb{C}}_{\lambda}$. It is important to note and to memorize that if we identify $\left|a_{1}\right|$ with $\left|a_{4}\right|$ and also $\left|a_{2}\right|$ with $\left|a_{3}\right|$ in $C_{\lambda} \backslash|a|$, then the resulting surface is nothing but $\widehat{\mathbb{C}}_{\mu}$.

Next we examine another simply connected region $\widehat{\mathbb{C}}_{\mu} \backslash \pi_{\mu}^{-1}([\lambda, \mu])$. The fiber $\pi_{\mu}^{-1}(\mu)$ is the one point set in $\widehat{\mathbb{C}}_{\mu}$, the point of which is denoted by $\mu_{0}$. The point $\mu_{0}$ is the branch point of multiplicity 2 of the covering surface $\left(\widehat{\mathbb{C}}_{\mu}, \widehat{\mathbb{C}}, \pi_{\mu}\right)$. Recall that $\widehat{\mathbb{C}}_{\mu} \backslash \pi_{\mu}^{-1}(\gamma(\mu))$ consists of two sheets, the upper sheets $\widehat{\mathbb{C}}_{1} \backslash \gamma(\mu)$ and the lower sheet $\widehat{\mathbb{C}}_{2} \backslash \gamma(\mu)$. We denote by $[\lambda, \mu]_{j}$ the part over $[\lambda, \mu]$ of the slit $\gamma(\mu)$ in $\widehat{\mathbb{C}}_{j} \backslash \gamma(\mu)(j=1,2)$ and by $[\lambda, \mu]_{j}^{+}$and $[\lambda, \mu]_{j}^{-}$the upper and the lower edge of $[\lambda, \mu]_{j}$. Then the fiber $\pi_{\mu}(\lambda)^{-1}$ is the two points set consisting of the end point $\lambda_{1}$ over $\lambda$ of $[\lambda, \mu]_{1}^{+}=[\lambda, \mu]_{2}^{-}$and the end point $\lambda_{2}$ over $\lambda$ of $[\lambda, \mu]_{1}^{-}=[\lambda, \mu]_{2}^{+}$. We then set $b_{1}=b_{1}(\mu):=\pi_{\mu}^{-1}([\lambda, \mu]) \cap[\lambda, \mu]_{1}^{+}$, 
$b_{2}=b_{2}(\mu):=\pi_{\mu}^{-1}([\lambda, \mu]) \cap[\lambda, \mu]_{1}^{-}, b_{3}=b_{3}(\mu):=\pi_{\mu}^{-1}([\lambda, \mu]) \cap[\lambda, \mu]_{2}^{+}$, and $b_{4}=b_{4}(\mu):=\pi_{\mu}^{-1}([\lambda, \mu]) \cap[\lambda, \mu]_{2}^{-}$. As subsets of $\widehat{\mathbb{C}}_{\mu}$ we have

$$
\left|b_{1}\right|=\left|b_{4}\right|, \quad\left|b_{2}\right|=\left|b_{3}\right| \text {. }
$$

We consider $b_{1}$ and $b_{4}$ as directed line segments starting from $\lambda_{1}$ and terminating at $\mu_{0}$ and also $b_{2}$ and $b_{3}$ as directed line segments starting from $\lambda_{2}$ and terminating at $\mu_{0}$. Then

$$
b=b(\mu):=b_{1}-b_{2}+b_{3}-b_{4}
$$

is a Jordan curve which is the Carathéodory boundary of the Carathéodory compactification of $\widehat{\mathbb{C}}_{\mu} \backslash \pi_{\mu}^{-1}([\lambda, \mu])$. The curve $b$ is positively oriented and $b \backslash\left\{\lambda_{1}, \lambda_{2}\right\}$ is analytic and $b$ has two corner points at $\lambda_{1}$ and $\lambda_{2}$.

\section{$\S 5$. Standard local parameter at the branch point}

Recall that $\lambda_{0}$ is the branch point of multiplicity 2 of the covering surface $\widehat{\mathbb{C}}_{\lambda}$ over $\lambda \in \mathbb{C}$. We denote by $\Delta_{2}\left(\lambda_{0}, 4 r^{2}\right)$ the two sheeted disc with radius $4 r^{2}$ centered at $\lambda_{0}$, i.e. the set of points in $\widehat{\mathbb{C}}_{\lambda}$ lying over the $\operatorname{disc} \Delta\left(\lambda, 4 r^{2}\right):=\left\{z \in \mathbb{C}:|z-\lambda|<4 r^{2}\right\} \subset \widehat{\mathbb{C}}$. The local coordinate $\left(\Delta(0,2 r), \zeta_{\lambda}\right)$ at $\lambda_{0}$ given by

$$
\zeta_{\lambda}=\xi_{\lambda}+i \eta_{\lambda}:=\sqrt{z-\lambda} \quad(\sqrt{1}=1)
$$

is referred to as the standard local parameter at the branch point $\lambda_{0}$, where $\zeta_{\lambda} \in \mathbb{C}$ with $\left|\zeta_{\lambda}\right|<2 r$ and $\xi_{\lambda}=\Re \zeta_{\lambda}$ and $\eta_{\lambda}=\Im \zeta_{\lambda}$. Let $c_{j} \subset \Delta(0,2 r)$ be the image of $a_{j}$ by the conformal mapping of $\Delta_{2}\left(\lambda_{0}, 4 r^{2}\right)$ onto $\Delta(0,2 r)$ given by $(5.1)(j=1,2,3,4)$. Then $c_{1}$ and $c_{2}$ are directed straight line segments starting from 0 and terminating at $\sqrt{\mu-\lambda}$ (the positive square root of $\mu-\lambda>0)$ and $c_{3}$ and $c_{4}$ are directed straight line segments starting from 0 and terminating at $-\sqrt{\mu-\lambda}$. Considering $c_{1}$ and $c_{2}$ as the upper and the lower edge of $[0, \sqrt{\mu-\lambda}]$, and also $c_{3}$ and $c_{4}$ as the lower and the upper edge of $[0,-\sqrt{\mu-\lambda}]$, the curve

$$
c:=c_{1}-c_{2}+c_{3}-c_{4}
$$

is the image of $a$ which can be viewed as a Jordan curve in the sense of Carathéodory. Thus the support $|c|$ of $c$ is given, on setting $\varepsilon:=\sqrt{\mu-\lambda}>0$, by

$$
|c|=\left\{\zeta_{\lambda}=\xi_{\lambda}+i \eta_{\lambda} \in \Delta(0,2 r):\left|\zeta_{\lambda}\right| \leq \varepsilon, \eta_{\lambda}=0\right\}
$$


Similarly as above recall that $\mu_{0}$ is the branch point of multiplicity 2 of the covering surface $\widehat{\mathbb{C}}_{\mu}$ over $\mu \in \mathbb{C}$. The standard local parameter at $\mu_{0}$ valid on $\Delta(0,2 r)$ for $\Delta_{2}\left(\mu_{0}, 4 r^{2}\right)$ is

$$
\zeta_{\mu}=\xi_{\mu}+i \eta_{\mu}:=\sqrt{z-\mu} \quad(\sqrt{1}=1),
$$

where $\zeta_{\mu} \in \mathbb{C}$ with $\left|\zeta_{\mu}\right|<2 r$ and $\xi_{\mu}=\Re \zeta_{\mu}$ and $\eta_{\mu}=\Im \zeta_{\mu}$. Let $d_{j} \subset \Delta(0,2 r)$ be the image of $b_{j}$ by the conformal mapping of $\Delta_{2}\left(\mu_{0}, 4 r^{2}\right)$ onto $\Delta(0,2 r)$ given by (5.3). Then $d_{1}$ and $d_{4}$ are the directed straight line segments starting from $i \sqrt{\mu-\lambda}(i=\sqrt{-1})$ and terminating at 0 and also $d_{2}$ and $d_{3}$ are the directed straight line segments starting from $-i \sqrt{\mu-\lambda}$ and terminating at 0 . By considering $d_{1}$ and $d_{4}$ as the right and the left edge of $[i \sqrt{\mu-\lambda}, 0]$ and $d_{2}$ and $d_{3}$ are the right and the left edge of $[-i \sqrt{\mu-\lambda}, 0]$, the curve

$$
d:=d_{1}-d_{2}+d_{3}-d_{4}
$$

is the image of $b$ which can be viewed as a Jordan curve in the sense of Carathéodory. The support $|d|$ of $d$ is given by

$$
|d|=\left\{\zeta_{\mu}=\xi_{\mu}+i \eta_{\mu} \in \Delta(0,2 r): \xi_{\mu}=0,\left|\eta_{\mu}\right| \leq \varepsilon\right\}
$$

Pick an arbitrary Carathéodory boundary point $p$ of the surface $W$. We may view that $p \in a$ by understanding that $W=\widehat{\mathbb{C}}_{\lambda} \backslash \pi_{\lambda}^{-1}([\lambda, \mu])$ and at the same time $p \in b$ by understanding $W=\widehat{\mathbb{C}}_{\mu} \backslash \pi_{\mu}^{-1}([\lambda, \mu])$. Let $\zeta_{\lambda}$ be the coordinate of $p$ when we consider $p \in a$ and $\zeta_{\mu}$ be the coordinate of $p$ when we consider $p \in b$. It is important to know the relation between $\zeta_{\lambda}$ and $\zeta_{\mu}$. Suppose $p$ is lying over $z \in \mathbb{C}$. Then from $\sqrt{z-\lambda}=\zeta_{\lambda}$ and $\sqrt{z-\mu}=\zeta_{\mu}$ it follows that

$$
z=\lambda+\zeta_{\lambda}^{2}=\mu+\zeta_{\mu}^{2}
$$

Recall that we have set $\varepsilon=\sqrt{\mu-\lambda}$ (the positive square root of $\mu-\lambda>0$ ). Thus we obtain

$$
\zeta_{\lambda}^{2}=\varepsilon^{2}+\zeta_{\mu}^{2}
$$

Compareing the real and imaginary parts of both sides of the above we see that

$$
\left\{\begin{array}{l}
\xi_{\lambda}^{2}-\eta_{\lambda}^{2}=\varepsilon^{2}+\left(\xi_{\mu}^{2}-\eta_{\mu}^{2}\right), \\
\xi_{\lambda} \eta_{\lambda}=\xi_{\mu} \eta_{\mu} .
\end{array}\right.
$$

Since $\eta_{\lambda}=0$ on $c$ and $\xi_{\mu}=0$ on $d$, the coordinate transformation formula on the Carathéodory boundary of $W$ takes the following form:

$$
\xi_{\lambda}^{2}=\varepsilon^{2}-\eta_{\mu}^{2}
$$


Observe that $c_{1} \cup c_{2}$ corresponds to $d_{1} \cup d_{2}$ and $c_{3} \cup c_{4}$ to $d_{3} \cup d_{4}$ and $\xi_{\lambda} \geq 0$ on $c_{1} \cup c_{2}$ which occurs when $\eta_{\mu} \in d_{1} \cup d_{2}$ and $\xi_{\lambda} \leq 0$ on $c_{3} \cup c_{4}$ which occurs when $\eta_{\mu} \in d_{3} \cup d_{4}$. Hence on introducing the function $\delta_{\mu}\left(\eta_{\mu}\right)$ of $\eta_{\mu}$ given by

$$
\delta_{\mu}\left(\eta_{\mu}\right)= \begin{cases}+1 & \left(\eta_{\mu} \in d_{1} \cup d_{2}\right), \\ -1 & \left(\eta_{\mu} \in d_{3} \cup d_{4}\right),\end{cases}
$$

we can express $\xi_{\lambda}$ in terms of $\eta_{\mu}$ :

$$
\xi_{\lambda}=\delta_{\mu}\left(\eta_{\mu}\right) \sqrt{\varepsilon^{2}-\eta_{\mu}^{2}}
$$

where $\sqrt{\varepsilon^{2}-\eta_{\mu}^{2}}$ is the positive square root of $\varepsilon^{2}-\eta_{\mu}^{2} \geq 0$. Similarly as above observe that $d_{1} \cup d_{2}$ corresponds to $c_{1} \cup c_{2}$ and $d_{3} \cup d_{4}$ to $c_{3} \cup c_{4}$, and $\eta_{\mu} \geq 0$ on $d_{1} \cup d_{4}$ which occurs when $\xi_{\lambda} \in c_{1} \cup c_{4}$ and $\eta_{\mu} \leq 0$ on $d_{2} \cup d_{3}$ which occurs when $\xi_{\lambda} \in c_{2} \cup c_{3}$. Thus on introducing the function $\delta_{\lambda}\left(\xi_{\lambda}\right)$ of $\xi_{\lambda}$ by

$$
\delta_{\lambda}\left(\xi_{\lambda}\right)= \begin{cases}+1 & \left(\xi_{\lambda} \in c_{1} \cup c_{4}\right), \\ -1 & \left(\xi_{\lambda} \in c_{2} \cup c_{3}\right)\end{cases}
$$

we can express $\eta_{\mu}$ in terms of $\xi_{\lambda}$ :

$$
\eta_{\mu}=\delta_{\lambda}\left(\xi_{\lambda}\right) \sqrt{\varepsilon^{2}-\xi_{\lambda}^{2}}
$$

where $\sqrt{\varepsilon^{2}-\xi_{\lambda}^{2}}$ is the positive square root of $\varepsilon^{2}-\xi_{\lambda}^{2} \geq 0$. Thus (5.5) gives rise to two formulas $(5.7)$ and $(5.9)$ but just one relation in the differential form

$$
\xi_{\lambda} d \xi_{\lambda}=-\eta_{\mu} d \eta_{\mu}
$$

\section{§. The harmonic version of the Cauchy estimates}

In the calculations with estimates performed in the next two succeeding sections we need to use the Cauchy estimates for harmonic functions (cf. e.g. [1]). Thus it will be convenient to recall their exact formulations in the form suitable for our later use, which will be briefly described in this short section. 
For each couple $(m, n)$ of nonnegative integers $m$ and $n$, we consider the finite positive quantity $C(m, n)$ determined only by the couple $(m, n)$ given by

$$
C(m, n):=\frac{1}{2 \pi} \int_{|\zeta|=1}\left|\left[\frac{\partial^{m+n}}{\partial x^{m} \partial y^{n}} \Re\left(\frac{\zeta+z}{\zeta-z}\right)\right]_{z=0}\right||d \zeta|,
$$

where $z=x+i y \in \mathbb{C}, \zeta \in \mathbb{C}$ with $|\zeta|=1$, and $|d \zeta|=d \theta$ with $\zeta=e^{i \theta}$. If $h \in H(\Delta(\alpha, r))$ with $|h| \leq M$ on $\Delta(\alpha, r)$, then we have the basic Cauchy estimates for harmonic functions:

$$
\left|\frac{\partial^{m+n}}{\partial x^{m} \partial y^{n}} h(\alpha)\right| \leq\left(C(m, n) / r^{m+n}\right) M
$$

for each pair $(m, n)$ of nonnegative integers $m$ and $n$, where $\alpha \in \mathbb{C}$ and $0<r<+\infty$, and $H(X)$ is the class of harmonic functions on an open subset $X$ of a Riemann surface, and $M$ is a finite positive constant. For a proof we use the Poisson representation formula of the harmonic function $w \mapsto h(\alpha+s w)$ defined for $|w| \leq 1$, where $s$ is an arbitrarily fixed real number with $0<s<r$ :

$$
h(\alpha+s w)=\frac{1}{2 \pi} \int_{0}^{2 \pi} h(\alpha+s \zeta) \Re\left(\frac{\zeta+w}{\zeta-w}\right) d \theta \quad\left(\zeta=e^{i \theta}\right) .
$$

Let $z=s w$ with $z=x+i y$ and $w=u+i v$. Then we see that

$$
\frac{\partial^{m+n}}{\partial u^{m} \partial v^{n}} h(\alpha+s w)=s^{m+n} \frac{\partial^{m+n}}{\partial x^{m} \partial y^{n}} h(\alpha+z),
$$

which implies that

$$
\begin{aligned}
& s^{m+n}\left|\frac{\partial^{m+n}}{\partial x^{m} \partial y^{n}} h(\alpha)\right|=\left|\frac{\partial^{m+n}}{\partial u^{m} \partial v^{n}} h(\alpha)\right| \\
& \quad=\frac{1}{2 \pi}\left|\int_{0}^{2 \pi} h(\alpha+s \zeta)\left[\frac{\partial^{m+n}}{\partial u^{m} \partial v^{n}} \Re\left(\frac{\zeta+w}{\zeta-w}\right)\right]_{w=0} d \theta\right| \\
& \quad \leq \frac{1}{2 \pi} \int_{0}^{2 \pi}|h(\alpha+s \zeta)|\left[\frac{\partial^{m+n}}{\partial x^{m} \partial y^{n}} \Re\left(\frac{\zeta+z}{\zeta-z}\right)\right]_{z=0} \mid d \theta \\
& \quad \leq \frac{1}{2 \pi} \int_{0}^{2 \pi} M\left|\left[\frac{\partial^{m+n}}{\partial x^{m} \partial y^{n}} \Re\left(\frac{\zeta+z}{\zeta-z}\right)\right]_{z=0}\right| d \theta=C(m, n) M,
\end{aligned}
$$

which implies the desired (6.2) with $r$ replaced by $s$. Since $0<s<r$ is arbitrary, on letting $s \uparrow r$, we deduce the genuine (6.2). The following form 
of the Cauchy estimates for harmonic functions will be extensively made use of in the next subsequent two sections: if $u \in H(\Delta(0,2 r))(0<r<+\infty)$, then we have

$$
\sup _{z \in \Delta(0, r)}\left|\frac{\partial^{m+n}}{\partial x^{m} \partial y^{n}} u(z)\right| \leq\left(C(m, n) / r^{m+n}\right) \sup _{z \in \Delta(0,2 r)}|u(z)|
$$

for any pair $(m, n)$ of nonnegative integers $m$ and $n$. For a proof, let $M:=$ $\sup _{z \in \Delta(0,2 r)}|u(z)|>0$. If $M=+\infty$, then (6.3) holds trivially. Thus suppose that $M<+\infty$ and take an arbitrary $z \in \Delta(0, r)$. Then, since $\Delta(z, r) \subset$ $\Delta(0,2 r)$, we have $|u| \leq M$ on $\Delta(z, r)$ and hence by (6.2) we see that

$$
\left|\frac{\partial^{m+n}}{\partial x^{m} \partial y^{n}} u(z)\right| \leq\left(C(m, n) / r^{m+n}\right) M
$$

for any $z \in \Delta(0, r)$, from which (6.3) follows instantly.

\section{§7. Calculation of $D\left(u_{\lambda}-u_{\mu}, u_{\lambda}\right)$}

As we saw in Section 3 we only have to prove (4.1) in order to complete the proof of Theorem 2.1. For the purpose we need to compute

$$
\lim _{\mu \downarrow \lambda} \frac{D\left(u_{\mu}\right)-D\left(u_{\lambda}\right)}{\mu-\lambda} .
$$

By the identity (4.3), the above computation will be achieved if we can finish the computations of the following two limits:

$$
\lim _{\mu \downarrow \lambda} \frac{D\left(u_{\lambda}-u_{\mu}, u_{\lambda}\right)}{\mu-\lambda}
$$

and

$$
\lim _{\mu \downarrow \lambda} \frac{D\left(u_{\mu}-u_{\lambda}, u_{\mu}\right)}{\mu-\lambda}
$$

In this section we compute $D\left(u_{\lambda}-u_{\mu}, u_{\lambda}\right)$ with estimate for the purpose to study (7.1) and those for (7.2) will be done in the next section.

By the Green formula we see that

$$
D\left(u_{\lambda}-u_{\mu}, u_{\lambda}\right)=\int_{a}\left(u_{\lambda}-u_{\mu}\right) * d u_{\lambda}
$$


Analyzing the behavior of $u_{\lambda}$ and $* d u_{\lambda}$ on $a_{1}-a_{2}$ and $a_{3}-a_{4}$ we see that

$$
\int_{a} u_{\lambda} * d u_{\lambda}=\int_{a} k * d u_{\lambda}=0
$$

for every constant $k \in \mathbb{R}$ (the set of real numbers) and therefore we deduce in particular

$$
D\left(u_{\lambda}-u_{\mu}, u_{\lambda}\right)=-\int_{a}\left(u_{\mu}-u_{\mu}\left(\mu_{0}\right)\right) * d u_{\lambda} .
$$

Recall that $\left(\Delta(0,2 r), \zeta_{\lambda}=\xi_{\lambda}+i \eta_{\lambda}\right)$ is a fixed local coordinate at the two sheeted disc $\Delta_{2}\left(\lambda_{0}, 4 r^{2}\right)$. Since $u_{\lambda} \in H\left(\Delta_{2}\left(\lambda_{0}, 4 r^{2}\right)\right)$, the coordinate expression of the differential $* d u_{\lambda}$ takes the form

$$
* d u_{\lambda}\left(\zeta_{\lambda}\right)=-\left(u_{\lambda}\right)_{\eta_{\lambda}}\left(\zeta_{\lambda}\right) d \xi_{\lambda}+\left(u_{\lambda}\right)_{\xi_{\lambda}} d \eta_{\lambda}
$$

where, for example, $\left(u_{\lambda}\right)_{\eta_{\lambda}}=\partial u_{\lambda} / \partial \eta_{\lambda}$. We have denoted by $c \subset \Delta(0,2 r)$ the image of $a \subset \Delta_{2}\left(\lambda_{0}, 4 r^{2}\right)$. Note that $\eta_{\lambda}=0$ on $c$. Hence

$$
* d u_{\lambda}\left(\zeta_{\lambda}\right)=* d u_{\lambda}\left(\xi_{\lambda}\right)=-\left(u_{\lambda}\right)_{\eta_{\lambda}}\left(\xi_{\lambda}\right) d \xi_{\lambda}
$$

on $c$. Hence from (7.3) it fillows that

$$
D\left(u_{\lambda}-u_{\mu}, u_{\lambda}\right)=\int_{c}\left(u_{\mu}\left(\xi_{\lambda}\right)-u_{\mu}\left(\mu_{0}\right)\right)\left(u_{\lambda}\right)_{\eta_{\lambda}}\left(\xi_{\lambda}\right) d \xi_{\lambda}
$$

By applying the Taylor expansion theorem (or simply the mean value theorem) we obtain

$$
\left(u_{\lambda}\right)_{\eta_{\lambda}}\left(\xi_{\lambda}\right)=\left(u_{\lambda}\right)_{\eta_{\lambda}}(0)+\left(u_{\lambda}\right)_{\eta_{\lambda} \xi_{\lambda}}\left(\theta_{1} \xi_{\lambda}\right) \xi_{\lambda} \quad\left(0<\theta_{1}<1\right)
$$

In view of $u_{\mu} \in H\left(\Delta_{2}\left(\mu_{0}, 4 r^{2}\right)\right)$, again by applying the Taylor expansion theorem using the local parameter $\zeta_{\mu}=i \eta_{\mu} \in d$ corresponding to $\zeta_{\lambda}=\xi_{\lambda} \in$ $c$, we deduce

$$
\begin{aligned}
u_{\mu}\left(\xi_{\lambda}\right)-u_{\mu}\left(\mu_{0}\right) & =u_{\mu}\left(i \eta_{\mu}\right)-u_{\mu}(0) \\
& =\left(u_{\mu}\right)_{\eta_{\mu}}(0) \eta_{\mu}+\frac{1}{2}\left(u_{\mu}\right)_{\eta_{\mu} \eta_{\mu}}\left(i \theta_{2} \eta_{\mu}\right) \eta_{\mu}^{2} \quad\left(0<\theta_{2}<1\right) .
\end{aligned}
$$

By (5.9) we obtain

$$
\begin{aligned}
& u_{\mu}\left(\xi_{\mu}\right)-u_{\mu}\left(\mu_{0}\right) \\
& \quad=\left(u_{\mu}\right)_{\eta_{\mu}}(0) \delta_{\lambda}\left(\xi_{\lambda}\right) \sqrt{\varepsilon^{2}-\xi_{\lambda}^{2}}+\frac{1}{2}\left(u_{\mu}\right)_{\eta_{\mu} \eta_{\mu}}\left(i \varepsilon_{2} \eta_{\mu}\right)\left(\varepsilon^{2}-\xi_{\lambda}^{2}\right)
\end{aligned}
$$


A fortiori we conclude that

$$
\begin{aligned}
& D\left(u_{\lambda}-u_{\mu}, u_{\lambda}\right) \\
& \quad=\left(u_{\lambda}\right)_{\eta_{\lambda}}(0)\left(u_{\mu}\right)_{\eta_{\mu}}(0) \int_{c} \delta_{\lambda}\left(\xi_{\lambda}\right) \sqrt{\varepsilon^{2}-\xi_{\lambda}^{2}} d \xi_{\lambda}+\int_{c} J\left(\xi_{\lambda}\right) d \xi_{\lambda},
\end{aligned}
$$

where

$$
\begin{aligned}
J\left(\xi_{\lambda}\right):=\left(u_{\mu}\left(\xi_{\lambda}\right)-u_{\mu}\left(\mu_{0}\right)\right)\left(u_{\lambda}\right)_{\eta_{\lambda}}\left(\xi_{\lambda}\right) & \\
& -\left(u_{\lambda}\right)_{\eta_{\lambda}}(0)\left(u_{\mu}\right)_{\eta_{\mu}}(0) \delta_{\lambda}\left(\xi_{\lambda}\right) \sqrt{\varepsilon^{2}-\xi_{\lambda}^{2}}
\end{aligned}
$$

Note that

$$
\begin{gathered}
\int_{c} \delta_{\lambda}\left(\xi_{\lambda}\right) \sqrt{\varepsilon^{2}-\xi_{\lambda}^{2}} d \xi_{\lambda}=\sum_{j=1}^{4} \int_{(-1)^{j-1} c_{j}} \delta_{\lambda}\left(\xi_{\lambda}\right) \sqrt{\varepsilon^{2}-\xi_{\lambda}^{2}} d \xi_{\lambda} \\
=4 \int_{0}^{\varepsilon} \sqrt{\varepsilon^{2}-\xi_{\lambda}^{2}} d \xi_{\lambda}=4 \varepsilon^{2} \int_{0}^{1} \sqrt{1-t^{2}} d t=\pi \varepsilon^{2} .
\end{gathered}
$$

Hence the first term on the right hand side of (7.7) is

$$
\pi\left(u_{\lambda}\right)_{\eta_{\lambda}}(0)\left(u_{\mu}\right)_{\eta_{\mu}}(0)(\mu-\lambda)
$$

Next we estimate the second term on the right hand side of (7.7). From (7.5) and (7.6) it follows that $J\left(\xi_{\lambda}\right)$ is

$$
\begin{aligned}
& \frac{1}{2}\left(u_{\lambda}\right)_{\eta_{\lambda}}(0)\left(u_{\mu}\right)_{\eta_{\mu} \eta_{\mu}}\left(i \theta_{2} \eta_{\mu}\right)\left(\varepsilon^{2}-\xi_{\lambda}^{2}\right) \\
& \quad+\left(u_{\lambda}\right)_{\eta_{\lambda} \eta_{\lambda}}\left(\theta_{1} \xi_{\lambda}\right)\left(u_{\mu}\right)_{\eta_{\mu}}(0) \delta_{\lambda}\left(\xi_{\lambda}\right) \xi_{\lambda} \sqrt{\varepsilon^{2}-\xi_{\lambda}^{2}} \\
& \quad+\frac{1}{2}\left(u_{\lambda}\right)_{\eta_{\lambda} \xi_{\lambda}}\left(\theta_{1} \xi_{\lambda}\right)\left(u_{\mu}\right)_{\eta_{\mu} \eta_{\mu}}\left(i \theta_{2} \eta_{\mu}\right) \xi_{\lambda}\left(\varepsilon^{2}-\xi_{\lambda}^{2}\right)
\end{aligned}
$$

Restricting $\xi_{\lambda}$ to $0 \leq \xi_{\lambda}<1$, we have $\varepsilon^{2}-\xi_{\lambda}^{2} \leq \varepsilon^{2},\left|\xi_{\lambda}\right| \sqrt{\varepsilon^{2}-\xi_{\lambda}^{2}} \leq \varepsilon^{2}$, and $\left|\xi_{\lambda}\right|\left(\varepsilon^{2}-\xi_{\lambda}^{2}\right) \leq \varepsilon^{3}<\varepsilon^{2}$. Since $\left|u_{\lambda}\right| \leq 1$ and $\left|u_{\mu}\right| \leq 1$, an application of (6.3) implies that

$$
\begin{aligned}
\left|J\left(\xi_{\lambda}\right)\right| & \leq\left(\frac{1}{2} C(0,2) C(0,1)+C(0,1) C(1,1)+\frac{1}{2} C(1,1) C(0,2)\right) \frac{\varepsilon^{2}}{r^{3}} \\
& =: \frac{C_{1}}{4 r^{3}} \varepsilon^{2}
\end{aligned}
$$


so that we obtain

$$
\begin{aligned}
\left|\int_{c} J\left(\xi_{\lambda}\right) d \xi_{\lambda}\right| & =\left|\sum_{j=1}^{4} \int_{(-1)^{j-1} c_{j}} J\left(\xi_{\lambda}\right) d \xi_{\lambda}\right| \leq \sum_{j=1}^{4}\left|\int_{c_{j}}\right| J\left(\xi_{\lambda}\right)\left|d \xi_{\lambda}\right| \\
& \leq 4 \int_{0}^{\varepsilon} \frac{C_{1}}{4 r^{3}} \varepsilon^{2} d \xi_{\lambda}=\frac{C_{1}}{r^{3}} \varepsilon^{3}=\frac{C_{1}}{r^{3}}(\mu-\lambda)^{3 / 2}
\end{aligned}
$$

and finally we come to the estimate

$$
\left|D\left(u_{\lambda}-u_{\mu}, u_{\lambda}\right)-\pi\left(u_{\lambda}\right)_{\eta_{\lambda}}(0)\left(u_{\mu}\right)_{\eta_{\mu}}(0)(\mu-\lambda)\right| \leq \frac{C_{1}}{r^{3}}(\mu-\lambda)^{3 / 2}
$$

where we stress here that $C_{1}$ is, by its definition above, a universal constant and in particular does not depend upon $\mu$.

\section{§8. Calculation of $D\left(u_{\mu}-u_{\lambda}, u_{\mu}\right)$}

The computation of the mutual Dirichlet integral $D\left(u_{\mu}-u_{\lambda}, u_{\mu}\right)$ is almost identical with that of $D\left(u_{\lambda}-u_{\mu}, u_{\lambda}\right)$ performed in the preceeding Section 7. By the Green formula

$$
D\left(u_{\mu}-u_{\lambda}, u_{\mu}\right)=\int_{b}\left(u_{\mu}-u_{\lambda}\right) * d u_{\mu}
$$

On analyzing the behavior of $u_{\mu}$ and $* d u_{\mu}$ on $b_{1}-b_{2}$ and also on $b_{3}-b_{4}$, we can deduce that

$$
\int_{b} u_{\mu} * d u_{\mu}=\int_{b} k * d u_{\mu}=0
$$

for any constant $k \in \mathbb{R}$. Therefore we see that

$$
D\left(u_{\mu}-u_{\lambda}, u_{\mu}\right)=-\int_{b}\left(u_{\lambda}-u_{\lambda}\left(\lambda_{0}\right)\right) * d u_{\mu} .
$$

Note that $u_{\mu} \in H\left(\Delta_{2}\left(\mu_{0}, 4 r^{2}\right)\right)$. We use the local coordinate $\left(\Delta(0,2 r), \zeta_{\mu}=\right.$ $\left.\xi_{\mu}+i \eta_{\mu}\right)$ at $\Delta\left(\mu_{0}, 4 r^{2}\right)$ to have the coordinate expression

$$
* d u_{\mu}\left(\zeta_{\mu}\right)=-\left(u_{\mu}\right)_{\eta_{\mu}} d \xi_{\mu}+\left(u_{\mu}\right)_{\xi_{\mu}} d \eta_{\mu}
$$

of $* d u_{\mu}$. Since $\xi_{\mu}=0$ on the image $d$ of $b$, we have

$$
* d u_{\mu}\left(\xi_{\mu}\right)=* d u_{\mu}\left(i \eta_{\mu}\right)=\left(u_{\mu}\right)_{\xi_{\mu}}\left(i \eta_{\mu}\right) d \eta_{\mu}
$$

on $d$. Thus

$$
D\left(u_{\mu}-u_{\lambda}, u_{\mu}\right)=-\int_{d}\left(u_{\lambda}\left(i \eta_{\mu}\right)-u_{\lambda}\left(\lambda_{0}\right)\right)\left(u_{\mu}\right)_{\xi_{\mu}}\left(i \eta_{\mu}\right) d \eta_{\mu}
$$


By the Taylor expansion theorem (or rather the mean value theorem), we have

$$
\left(u_{\mu}\right)_{\xi_{\mu}}\left(i \eta_{\mu}\right)=\left(u_{\mu}\right)_{\xi_{\mu}}(0)+\left(u_{\mu}\right)_{\xi_{\mu}} \eta_{\mu}\left(i \theta_{1} \eta_{\mu}\right) \eta_{\mu} \quad\left(0<\theta_{1}<1\right) .
$$

Concerning the other factor $u_{\lambda}\left(i \eta_{\mu}\right)-u_{\lambda}\left(\lambda_{0}\right)$ of the integrand in (8.1), since $u_{\lambda} \in H\left(\Delta_{2}\left(\lambda_{0}, 4 r^{2}\right)\right)$, by using $\zeta_{\mu}=\xi_{\lambda} \in c$ corresponding to $\zeta_{\mu}=i \eta_{\mu} \in d$ and the Taylor expansion theorem, we obtain

$$
\begin{aligned}
u_{\lambda}\left(i \eta_{\mu}\right)-u_{\lambda}\left(\lambda_{0}\right) & =u_{\lambda}\left(\xi_{\lambda}\right)-u_{\lambda}(0) \\
& =\left(u_{\lambda}\right)_{\xi_{\lambda}}(0) \xi_{\lambda}+\frac{1}{2}\left(u_{\lambda}\right)_{\xi_{\lambda} \xi_{\lambda}}\left(\theta_{2} \xi_{\lambda}\right) \xi_{\lambda}^{2} \quad\left(0<\theta_{2}<1\right) .
\end{aligned}
$$

Hence by using (5.8) we see that

$$
\begin{aligned}
u_{\lambda}\left(i \eta_{\mu}\right)-u_{\lambda}\left(\lambda_{0}\right)=\left(u_{\lambda}\right)_{\xi_{\lambda}}(0) \delta_{\eta_{\mu}}\left(\eta_{\mu}\right) \sqrt{\varepsilon^{2}-\eta_{\mu}^{2}} & \\
& +\frac{1}{2}\left(u_{\lambda}\right)_{\xi_{\lambda} \xi_{\lambda}}\left(\theta_{2} \xi_{\lambda}\right)\left(\varepsilon^{2}-\eta_{\mu}^{2}\right)
\end{aligned}
$$

Therefore we have

$$
\begin{gathered}
D\left(u_{\mu}-u_{\lambda}, u_{\mu}\right)=-\left(u_{\lambda}\right)_{\xi_{\lambda}}(0)\left(u_{\mu}\right)_{\xi_{\mu}}(0) \int_{d} \delta_{\mu}\left(\eta_{\mu}\right) \sqrt{\varepsilon^{2}-\eta_{\mu}^{2}} d \eta_{\mu} \\
-\int_{d} J\left(\eta_{\mu}\right) d \eta_{\mu}
\end{gathered}
$$

where

$$
\begin{aligned}
J\left(\eta_{\mu}\right):=\left(u_{\lambda}\left(i \eta_{\mu}\right)-u_{\lambda}\left(\lambda_{0}\right)\right)\left(u_{\mu}\right)_{\xi_{\mu}}\left(i \eta_{\mu}\right) \\
-\left(u_{\lambda}\right) \xi_{\lambda}(0)\left(u_{\mu}\right) \xi_{\mu}(0) \delta_{\mu}\left(\eta_{\mu}\right) \sqrt{\varepsilon^{2}-\eta_{\mu}^{2}}
\end{aligned}
$$

Since

$$
\begin{gathered}
\int_{d} \delta_{\mu}\left(\eta_{\mu}\right) \sqrt{\varepsilon^{2}-\eta_{\mu}^{2}} d \eta_{\mu}=\sum_{j=1}^{4} \int_{(-1)^{j-1} d_{j}} \delta_{\mu}\left(\eta_{\mu}\right) \sqrt{\varepsilon^{2}-\eta_{\mu}^{2}} d \eta_{\mu} \\
=-4 \int_{0}^{\varepsilon} \sqrt{\varepsilon^{2}-\eta_{\mu}^{2}} d \eta_{\mu}=-4 \varepsilon^{2} \int_{0}^{1} \sqrt{1-t^{2}} d t=-\pi \varepsilon^{2}
\end{gathered}
$$

the first term of the right hand side of (8.4) is

$$
\pi\left(u_{\lambda}\right)_{\xi_{\lambda}}(0)\left(u_{\mu}\right)_{\xi_{\mu}}(0)(\mu-\lambda)
$$


Next we evaluate the second term of the right hand side of (8.4). By (8.2) and (8.3) we see that $J\left(\eta_{\mu}\right)$ is

$$
\begin{aligned}
& \frac{1}{2}\left(u_{\mu}\right)_{\xi_{\mu}}(0)\left(u_{\lambda}\right)_{\xi_{\lambda} \xi_{\lambda}}\left(i \theta_{2} \xi_{\lambda}\right)\left(\varepsilon^{2}-\eta_{\mu}^{2}\right) \\
& \quad+\left(u_{\mu}\right)_{\xi_{\mu} \eta_{\mu}}\left(i \theta_{1} \eta_{\mu}\right)\left(u_{\lambda}\right)_{\xi_{\lambda}}(0) \delta_{\mu}\left(\eta_{\mu}\right) \xi_{\lambda} \sqrt{\varepsilon^{2}-\xi_{\lambda}^{2}} \\
& \quad+\frac{1}{2}\left(u_{\mu}\right)_{\xi_{\mu} \eta_{\mu}}\left(i \theta_{1} \eta_{\mu}\right)\left(u_{\lambda}\right)_{\xi_{\lambda} \xi_{\lambda}}\left(\theta_{2} \xi_{\lambda}\right) \eta_{\mu}\left(\varepsilon^{2}-\eta_{\mu}^{2}\right)
\end{aligned}
$$

Observe that $\left|u_{\lambda}\right| \leq 1$ and $\left|u_{\mu}\right| \leq 1$. If we restrict $\eta_{\mu}$ to $0 \leq \eta_{\mu} \leq \varepsilon<1$, then $\varepsilon^{2}-\eta_{\mu}^{2} \leq \varepsilon^{2},\left|\eta_{\mu}\right| \sqrt{\varepsilon^{2}-\eta_{\mu}^{2}} \leq \varepsilon^{2}$, and $\left|\eta_{\mu}\right|\left(\varepsilon^{2}-\eta_{\mu}^{2}\right) \leq \varepsilon^{3} \leq \varepsilon^{2}$. Hence the inequality (6.3) implies that

$$
\begin{aligned}
\left|J\left(\eta_{\mu}\right)\right| & \leq\left(\frac{1}{2} C(2,0) C(1,0)+C(1,0) C(1,1)+\frac{1}{2} C(1,1) C(2,2)\right) \frac{\varepsilon^{2}}{r^{3}} \\
& =: \frac{C_{2}}{4 r^{3}} \varepsilon^{2} .
\end{aligned}
$$

Therefore we deduce

$$
\begin{aligned}
\left|\int_{d} J\left(\eta_{\mu}\right) d \eta_{\mu}\right| & =\left|\sum_{j=1}^{4} \int_{(-1)^{j-1} d_{j}} J\left(\eta_{\mu}\right) d \eta_{\mu}\right| \leq \sum_{j=1} 4\left|\int_{d_{j}}\right| J\left(\eta_{\mu}\right)\left|d \eta_{\mu}\right| \\
& \leq 4 \int_{0}^{\varepsilon} \frac{C_{2}}{4 r^{3}} \varepsilon^{2} d \eta_{\mu}=\frac{C_{2}}{r^{3}} \varepsilon^{3}=\frac{C_{2}}{r^{3}}(\mu-\lambda)^{3 / 2} .
\end{aligned}
$$

Thus the identity (8.4) implies that

$$
\left|D\left(u_{\mu}-u_{\lambda}, u_{\mu}\right)-\pi\left(u_{\lambda}\right)_{\xi_{\lambda}}(0)\left(u_{\mu}\right)_{\xi_{\mu}}(0)(\mu-\lambda)\right| \leq \frac{C_{2}}{r^{3}}(\mu-\lambda)^{3 / 2} .
$$

By (4.3), (7.8), and (8.5), we obtain, on setting $C:=C_{1}+C_{2}$, that

$$
\begin{aligned}
& \left|\left(D\left(u_{\mu}\right)-D\left(u_{\lambda}\right)\right)-\pi\left(\left(u_{\lambda}\right)_{\xi_{\lambda}}(0)\left(u_{\mu}\right)_{\xi_{\mu}}(0)-\left(u_{\lambda}\right)_{\eta_{\lambda}}(0)\left(u_{\mu}\right)_{\eta_{\mu}}(0)\right)(\mu-\lambda)\right| \\
& \quad \leq \frac{C}{r^{3}}(\mu-\lambda)^{3 / 2} .
\end{aligned}
$$

A fortiori we conclude that

$$
\begin{aligned}
& \lim _{\mu \downarrow \lambda} \frac{D\left(u_{\mu}\right)-D\left(u_{\lambda}\right)}{\mu-\lambda} \\
& \quad=\pi \lim _{\mu \downarrow \lambda}\left(\left(u_{\lambda}\right)_{\xi_{\lambda}}(0)\left(u_{\mu}\right)_{\xi_{\mu}}(0)-\left(u_{\lambda}\right)_{\eta_{\lambda}}(0)\left(u_{\mu}\right)_{\eta_{\mu}}(0)\right) .
\end{aligned}
$$

The calculation of the term on the right hand side of the above identity is the final task to complete the proof of (4.1) (i.e. (2.6)). 


\section{§9. Continuity of standard partial derivatives}

We denote by $\varphi_{\lambda}\left(\varphi_{\mu}\right.$, resp.) the conformal mapping $w=\varphi_{\lambda}\left(\zeta_{\lambda}\right)(w=$ $\varphi_{\mu}\left(\zeta_{\mu}\right)$, resp. $)$ of the coordinate neighborhood $\left(\Delta(0,2 r), \xi_{\lambda}=\xi_{\lambda}+i \eta_{\lambda}\right)$ $\left(\left(\Delta(0,2 r), \zeta_{\mu}=\xi_{\mu}+i \eta_{\mu}\right)\right.$, resp. $)$ onto the two sheeted disc $\Delta_{2}\left(\lambda_{0}, 4 r^{2}\right)$ $\left(\Delta_{2}\left(\mu_{0}, 4 r^{2}\right)\right.$, resp. $)$ with $\varphi_{\lambda}(0)=\lambda_{0}\left(\varphi_{\mu}(0)=\mu_{0}\right.$, resp. $)$ so that $\varphi_{\lambda}^{-1}\left(\varphi_{\mu}^{-1}\right.$, resp.) is the local parameter at $\lambda_{0}$ ( $\mu_{0}$, resp.). Recall that $\pi_{\lambda}\left(\pi_{\mu}\right.$, resp.) is the projection of $\Delta_{2}\left(\lambda_{0}, 4 r^{2}\right)\left(\Delta_{2}\left(\mu_{0}, 4 r^{2}\right)\right.$, resp. $)$ onto $\Delta\left(\lambda, 4 r^{2}\right)\left(\Delta\left(\mu, 4 r^{2}\right)\right.$, resp.).

Hereafter we consider only those $\mu>\lambda$ so close to $\lambda$ as $\mu-\lambda=: \varepsilon^{2}<$ $r^{2} / 16$. Then we fix $\rho \in(2 r, \lambda-\sigma)$ such that the closure of $\pi_{\lambda}^{-1}\left(\Delta\left(\lambda, \rho^{2}\right)\right)=$ $\pi_{\mu}^{-1}\left(\Delta\left(\lambda, \rho^{2}\right)\right)$ is disjoint from $A \cup B$, which is possible by the choice of $r$ in Section 4 at the very begining. Observe that

$$
S:=\pi_{\lambda}^{-1}\left(\Delta\left(\lambda, \rho^{2}\right) \backslash \bar{\Delta}\left(\lambda, \rho^{2} / 4\right)\right),
$$

$\bar{\Delta}\left(\lambda, \rho^{2} / 4\right)$ being the closure of $\Delta\left(\lambda, \rho^{2} / 4\right)$, is an annulus which is a subregion of

$$
W=W_{\mu}:=\widehat{\mathbb{C}}_{\mu} \backslash \pi_{\mu}^{-1}([\lambda, \mu])=\widehat{\mathbb{C}}_{\lambda} \backslash \pi_{\lambda}^{-1}([\lambda, \mu]) .
$$

Note that we can find an annulus $X$ such that

$$
\partial \Delta_{2}\left(\lambda_{0}, r^{2}\right) \cup \partial \Delta_{2}\left(\mu_{0}, r^{2}\right) \subset X \subset \bar{X} \subset S .
$$

Based upon the way $\widehat{\mathbb{C}}_{\lambda}$ and $\widehat{\mathbb{C}}_{\mu}$ were constructed we can see the existence of a mapping $T=T_{\mu}$ of $\partial \Delta_{2}\left(\lambda_{0}, r^{2}\right)$ to $\partial \Delta_{2}\left(\mu_{0}, r^{2}\right)$ satisfying the following three conditions by rotating $\varphi_{\lambda}$ and $\varphi_{\mu}$ suitably if necessary:

( $\alpha) T: \partial \Delta_{2}\left(\lambda_{0}, r^{2}\right) \rightarrow \partial \Delta_{2}\left(\mu_{0}, r^{2}\right)$ is a homeomorphism;

$(\beta)$ for any $p \in \partial \Delta_{2}\left(\lambda_{0}, r^{2}\right), \pi_{\lambda}(p)-\lambda=\pi_{\mu}(T(p))-\mu$;

$(\lambda)$ for any $p \in \partial \Delta_{2}\left(\lambda_{0}, r^{2}\right), \varphi_{\lambda}^{-1}(p)=\varphi_{\mu}(T(p))$.

Then clearly we have that

$$
\lim _{\mu \downarrow \lambda} T_{\mu}=\mathrm{id}
$$

uniformly on $\partial \Delta_{2}\left(\lambda_{0}, r^{2}\right)$, where id. is the identity mapping.

We denote by $H_{1}(S)$ the class of harmonic functions $h$ on $S$ with $|h| \leq 1$ on $S$. For every pair $(p, q)$ of points $p$ and $q$ in $S$ we set

$$
d(p, q ; S)=d(p, q):=\sup _{h \in H_{1}(S)}|h(p)-h(q)|,
$$


which is clearly a metric on on $S$. When we view $S \subset \mathbb{C}=(\mathbb{C}, z=x+i y)$ and $S=\{z \in \mathbb{C}: c<|z|<1\}$, then $\Re z$ and $\Im z$ are contained in $H_{1}(S)$. Hence $d(p, q) \geq|\Re p-\Re q|$ and $|\Im p-\Im q|$ and hence $d(p, q) \geq(1 / \sqrt{2})|p-q|$. By the equicontinuity of the class $H_{1}(S)$ (cf. e.g. [16]) as a consequence of the uniform boundedness of $\nabla H_{1}(S)$ (cf. (6.3)), we see that $d(p, q) /|p-q|$ is bounded on the product $K_{1} \times K_{2}$ of two arbitrary compact subsets $K_{1}$ and $K_{2}$ of $S$. Therefore

$$
\frac{1}{\sqrt{2}} \leq \inf _{(p, q) \in K_{1} \times K_{2}} \frac{d(p, q)}{|p-q|} \leq \sup _{(p, q) \in K_{1} \times K_{2}} \frac{d(p, q)}{|p-q|}<+\infty .
$$

This proves that the topology on $S$ induced by $d$ (i.e. the metric space $(S, d)$ ) is identical with the relative plane topology of $S$. In particular we have

$$
\lim _{\mu \downarrow \lambda} \sup _{p \in \partial \Delta_{2}\left(\lambda_{0}, r\right)} d\left(p, T_{\mu}(p)\right)=0 .
$$

Next we maintain that

$$
\lim _{\mu \downarrow \lambda} \sup _{K}\left|u_{\mu}-u_{\lambda}\right|=0, \quad \text { and } \quad \lim _{\nu \uparrow \lambda} \sup _{K}\left|u_{\nu}-u_{\lambda}\right|=0
$$

for any compact subset $K$ of $\widehat{\mathbb{C}}_{\lambda} \backslash\left\{\lambda_{0}\right\}$. Since the proof for the former relation of the above can be almost verbatimely applied to that for the latter relation of the above, we only prove the first part of the above. Since the family $\left\{u_{\mu}\right.$ : $\mu \downarrow \lambda\}$ forms a normal family (cf. e.g. [16]), for any countable subsequence of the net $(\mu: \mu \downarrow \lambda)$ contains a subsequence $\left(\mu_{n}: 1 \leq n<+\infty\right)$ such that $\left(u_{\mu_{n}}\right)_{n \geq 1}$ converges to a continuous function $u$ uniformly on each compact subset of $\widehat{\mathbb{C}}_{\lambda} \backslash\left\{\lambda_{0}\right\}$. It is easily seen that $u \in C\left(\widehat{\mathbb{C}}_{\lambda} \backslash\left\{\lambda_{0}\right\}\right) \cap H\left(\widehat{\mathbb{C}}_{\lambda_{0}} \backslash\left(\left\{\lambda_{0}\right\} \cup\right.\right.$ $A \cup B)$ ) with $0 \leq u \leq 1$ on $\widehat{\mathbb{C}}_{\lambda} \backslash\left\{\lambda_{0}\right\}$ and $u \mid A=1$ and $u \mid B=0$. Then $u$ can be harmonically continued to $\left\{\lambda_{0}\right\}$ so that $u \in C\left(\widehat{\mathbb{C}}_{\lambda}\right) \cap H\left(\widehat{\mathbb{C}}_{\lambda} \backslash(A \cup B)\right)$. Since $u-u_{\lambda} \in C\left(\widehat{\mathbb{C}}_{\lambda}\right) \cap H\left(\widehat{\mathbb{C}}_{\lambda} \backslash(A \cup B)\right)$ and $u-u_{\lambda}=0$ on $A \cup B$, we must conclude that $u-u_{\lambda} \equiv 0$. This proves (the first part of) (9.2).

Viewing $u_{\lambda}$ and $u_{\mu}$ as functions on $\left(\Delta(0,2 r), \zeta_{\lambda}\right)$ and on $\left(\Delta(0,2 r), \zeta_{\mu}\right)$, respectively, and applying the Poisson representation formula to $u_{\lambda}$ and $u_{\mu}$ on $\Delta(0, r)$ (or more precisely on $\left(\Delta(0, r), \zeta_{\lambda}\right)$ and $\left(\Delta(0, r), \zeta_{\mu}\right)$, respectively), we obtain

$$
\begin{cases}u_{\lambda}\left(\zeta_{\lambda}\right)=\frac{1}{2 \pi} \int_{|\zeta|=r} u_{\lambda}(\zeta) \Re\left(\frac{\zeta+\zeta_{\lambda}}{\zeta-\zeta_{\lambda}}\right) \frac{1}{i \zeta} d \zeta & \left(\zeta_{\lambda} \in \Delta(0, r)\right), \\ u_{\mu}\left(\zeta_{\mu}\right)=\frac{1}{2 \pi} \int_{|\zeta|=r} u_{\mu}(\zeta) \Re\left(\frac{\zeta+\zeta_{\mu}}{\zeta-\zeta_{\mu}}\right) \frac{1}{i \zeta} d \zeta & \left(\zeta_{\mu} \in \Delta(0, r)\right) .\end{cases}
$$


Differentiating both sides of the first and the second identities of the above with respect to $\xi_{\lambda}$ and $\eta_{\lambda}$ and $\xi_{\mu}$ and $\eta_{\mu}$ and then setting $\zeta_{\lambda}=0$ and $\zeta_{\mu}=0$, respectively, we obtain

$$
\left\{\begin{array}{l}
\left(u_{\lambda}\right)_{\xi_{\lambda}}(0)=\frac{1}{\pi r} \int_{0}^{2 \pi} u_{\lambda}\left(r e^{i \theta}\right) \cos \theta d \theta \\
\left(u_{\mu}\right)_{\xi_{\mu}}(0)=\frac{1}{\pi r} \int_{0}^{2 \pi} u_{\mu}\left(r e^{i \theta}\right) \cos \theta d \theta \\
\left(u_{\lambda}\right)_{\eta_{\lambda}}(0)=\frac{1}{\pi r} \int_{0}^{2 \pi} u_{\lambda}\left(r e^{i \theta}\right) \sin \theta d \theta \\
\left(u_{\mu}\right)_{\eta_{\mu}}(0)=\frac{1}{\pi r} \int_{0}^{2 \pi} u_{\mu}\left(r e^{i \theta}\right) \sin \theta d \theta
\end{array}\right.
$$

Therefore

$$
\begin{aligned}
& \left|\left(u_{\mu}\right)_{\xi_{\mu}}(0)-\left(u_{\lambda}\right)_{\xi_{\lambda}}(0)\right|=\left|\frac{1}{\pi r} \int_{0}^{2 \pi}\left[u_{\mu}\left(r e^{i \theta}\right)-u_{\lambda}\left(r e^{i \theta}\right)\right] \cos \theta d \theta\right| \\
& \leq \frac{1}{\pi r} \int_{0}^{2 \pi}\left|u_{\mu}\left(r e^{i \theta}\right)-u_{\lambda}\left(r e^{i \theta}\right)\right| d \theta \leq \frac{2}{r} \sup _{\theta \in[0,2 \pi]}\left|u_{\mu}\left(r e^{i \theta}\right)-u_{\lambda}\left(r e^{i \theta}\right)\right| .
\end{aligned}
$$

Then the correspondence $r e^{i \theta} \leftrightarrow p$ by the mapping $r e^{i \theta}=\varphi_{\lambda}(p)$ : $\partial \Delta_{2}\left(\lambda_{0}, r^{2}\right) \rightarrow \partial \Delta(0, r)$ is bijective and thus

$$
\begin{aligned}
& \left|u_{\mu}\left(r e^{i \theta}\right)-u_{\lambda}\left(r e^{i \theta}\right)\right|=\left|u_{\mu}(T(p))-u_{\lambda}(p)\right| \\
& \quad \leq\left|u_{\mu}(T(p))-u_{\lambda}(T(p))\right|+\left|u_{\lambda}(T(p))-u_{\lambda}(p)\right| \\
& \quad \leq \sup _{X}\left|u_{\mu}-u_{\lambda}\right|+\sup _{p \in \partial \Delta_{2}\left(\lambda_{0}, r^{2}\right)} d(p, T(p) ; S) .
\end{aligned}
$$

The two terms on the right most hand side of the above tend to 0 as $\mu \downarrow \lambda$ by (9.3) and (9.1). By repeating the same argument to $\left|\left(u_{\mu}\right)_{\eta_{\mu}}(0)-\left(u_{\lambda}\right)_{\eta_{\lambda}}(0)\right|$, we deduce that

$$
\left\{\begin{array}{l}
\lim _{\mu \downarrow \lambda}\left(u_{\mu}\right)_{\xi_{\mu}}(0)=\left(u_{\lambda}\right)_{\xi_{\lambda}}(0), \\
\lim _{\mu \downarrow \lambda}\left(u_{\mu}\right)_{\eta_{\mu}}(0)=\left(u_{\lambda}\right)_{\eta_{\lambda}}(0) .
\end{array}\right.
$$

Only for the purpose to complete the proof of our main result, Theorem 2.1, it is sufficient to have the above (9.4). Nevertheless we need the following stronger version of the above (9.4) for the proof of Theorem 1.1 given in the 
next section. However, fixing $\mu$ instead of $\lambda$ and making $\lambda \uparrow \mu$ we can repeat the similar argument as in this section to derive (9.4). Then we obtain (9.4) with $\mu \downarrow \lambda$ replaced by $\mu \uparrow \lambda$. Hence we deduce the following generalization of $(9.4)$ :

$$
\left\{\begin{array}{l}
\lim _{\mu \rightarrow \lambda}\left(u_{\mu}\right)_{\xi_{\mu}}(0)=\left(u_{\lambda}\right)_{\xi_{\lambda}}(0), \\
\lim _{\mu \rightarrow \lambda}\left(u_{\mu}\right)_{\eta_{\mu}}(0)=\left(u_{\lambda}\right)_{\eta_{\lambda}}(0) .
\end{array}\right.
$$

Then the above (9.4) (and of course (9.5)) with (8.6) finally yields that

$$
\mathcal{D}_{\lambda, 0} D\left(u_{\lambda}\right)=\pi\left[\left(\left(u_{\lambda}\right)_{\xi_{\lambda}}(0)\right)^{2}-\left(\left(u_{\lambda}\right)_{\eta_{\lambda}}(0)\right)^{2}\right] .
$$

This with (4.2) assures the validity of (4.1), which completes the proof of our main result in this paper: Theorem 2.1 .

\section{$\S 10$. Proof of Theorem 1.1}

By the formula (2.5) in our main theorem 2.1, we see that

$$
\mathcal{D}_{z, \theta+\pi} \operatorname{cap}\left(A, \widehat{\mathbb{C}}_{\gamma, z} \backslash B\right)=-\mathcal{D}_{z, \theta} \operatorname{cap}\left(A, \widehat{\mathbb{C}}_{\gamma, z} \backslash B\right)
$$

since $\cos (\theta+\pi)=-\cos \theta$ and $\sin (\theta+\pi)=-\sin \theta$. Hence, in particular, on setting $\theta=0$ in the above identity we have

$$
\mathcal{D}_{z, \pi} \operatorname{cap}\left(A, \widehat{\mathbb{C}}_{\gamma, z} \backslash B\right)=-\mathcal{D}_{z, 0} \operatorname{cap}\left(A, \widehat{\mathbb{C}}_{\gamma, z} \backslash B\right)
$$

This shows that $(\partial / \partial x) \operatorname{cap}\left(A, \widehat{\mathbb{C}}_{\gamma, z} \backslash B\right)(z=x+i y)$ exists and

$$
\frac{\partial}{\partial x} \operatorname{cap}\left(A, \widehat{\mathbb{C}}_{\gamma, z} \backslash B\right)=\pi\left[\left(\frac{\partial}{\partial \xi} u_{\gamma, z}(0)\right)^{2}-\left(\frac{\partial}{\partial \eta} u_{\gamma, z}(0)\right)^{2}\right]
$$

where $\zeta=\xi+i \eta$ is the standard local parameter at the branch point $\tilde{z}$ in $\widehat{\mathbb{C}}_{\gamma, z} \backslash(A \cup B)$ lying over $z$. Moreover (9.5) assures that the right hand side of (10.2) is continuous in $z$. Similarly by setting $\theta=\pi / 2$ in (10.1) implies that

$$
\mathcal{D}_{z, \pi / 2+\pi} \operatorname{cap}\left(A, \widehat{\mathbb{C}}_{\gamma, z} \backslash B\right)=-\mathcal{D}_{z, \pi / 2} \operatorname{cap}\left(A, \widehat{\mathbb{C}}_{\gamma, z} \backslash B\right),
$$

which shows that $(\partial / \partial y) \operatorname{cap}\left(A, \widehat{\mathbb{C}}_{\gamma, z} \backslash B\right)(z=x+i y)$ exists and

$$
\frac{\partial}{\partial y} \operatorname{cap}\left(A, \widehat{\mathbb{C}}_{\gamma, z} \backslash B\right)=2 \pi\left(\frac{\partial}{\partial \xi} u_{\gamma, z}(0) \cdot \frac{\partial}{\partial \eta} u_{\gamma, z}(0)\right) .
$$


Again by (9.5) we see that the right hand side of (10.3) is continuous in $z$. In short, we have seen that the first order partial derivatives of $\operatorname{cap}\left(A, \widehat{\mathbb{C}}_{\gamma, z}\right)$ $B)$ exist and are continuous with respect to $z$ so that the function $z \mapsto$ $\operatorname{cap}\left(A, \widehat{\mathbb{C}}_{\gamma, z} \backslash B\right)$ considered only locally on $\mathbb{C}$ is of class $C^{1}$.

\section{REFERENCES}

[1] S. Axler, P. Bourdon and W. Ramey, Harmonic Function Theory, Second Edition, Springer, 2001.

[2] J. Heinonen, T. Kilpeläinen and O. Martio, Nonlinear Potential Theory of Degenerate Elliptic Equations, Oxford Univ. Press, 1993.

[3] N. Levenberg and H. Yamaguchi, The metric induced by the Robin function, Memoirs of the Amer. Math. Soc., 92\#448 (1991), 1-156.

[4] N. Levenberg and H. Yamaguchi, Robin functions for complex manifolds and applications, CR geometry and isolated singularities, Report of RIMS of Kyoto Univ., 1037 (1998), 138-142.

[5] N. Levenberg and H. Yamaguchi, Robin functions for complex manifolds and applications, Abstracts for lectures in Function Theory Symposium, 47 (2004), 26-47.

[6] M. Nakai, Types of complete infinitely sheeted planes, Nagoya Math. Jour., 176 (2004), 181-195.

[7] M. Nakai, Types of pasting arcs in two sheeted spheres, Potential Theory in Matsue, Advanced Studies in Pure Mathematics, 44 (2006), 291-304.

[8] M. Nakai, The role of compactification theory in the type problem, Hokkaido Math. Jour., to appear.

[9] M. Nakai and S. Segawa, Parabolicity of Riemann surfaces, Hokkaido Univ. Tech. Rep., Ser. in Math., 73 (2003), 111-116.

[10] M. Nakai and S. Segawa, A role of the completeness in the type problem for infinitely sheeted planes, Complex Variables, 49 (2004), 229-240.

[11] M. Nakai and S. Segawa, The role of symmetry for pasting arcs in the type problem, Complex Variables and Elliptic Equations, to appear.

[12] R. Nevannlina, Analytic Functions, Springer, 1970.

[13] H. E. Rauch, Weierstrass points, branch points, and the moduli of Riemann surfaces, Comm. Pure Appl. Math., 12 (1959), 543-560.

[14] B. Rodin and L. Sario, Principal Functions, Van Nostrand, 1970.

[15] L. Sario and M. Nakai, Classification Theory of Riemann Surfaces, Springer, 1970.

[16] J. L. Schiff, Normal Families, Springer, 1993.

[17] M. Tsuji, Potential Theory in Modern Function Theory, Maruzen, 1959.

[18] H. Yamaguchi, Sur le mouvement des constantes de Robin, J. Math. Kyoto Univ., 15 (1975), 53-71.

[19] H. Yamaguchi, Variations of pseudoconvex domains over $\mathbf{C}^{n}$, Michigan Math. Jour., 36 (1989), 415-457. 
Professor Emeritus AT:

Department of Mathematics

Nagoya Institute of Technology

Gokiso, Showa

Nagoya, 466-8555

Japan

MAILING AdDress:

52 Eguchi, Hinaga

Chita, 478-0041

Japan

nakai@daido-it.ac.jp 